\title{
Observing the Space- and Time-Dependent Growth of Correlations in Dynamically Tuned Synthetic Ising Models with Antiferromagnetic Interactions
}

\author{
Vincent Lienhard, Sylvain de Léséleuc, ${ }^{*}$ Daniel Barredo, Thierry Lahaye, and Antoine Browaeys \\ Laboratoire Charles Fabry, Institut d'Optique Graduate School, CNRS, \\ Université Paris-Saclay, F-91127 Palaiseau Cedex, France \\ Michael Schuler, ${ }^{*}$ Louis-Paul Henry, and Andreas M. Läuchli \\ Institut für Theoretische Physik, Universität Innsbruck, A-6020 Innsbruck, Austria
}

(Received 3 November 2017; revised manuscript received 17 March 2018; published 18 June 2018)

We explore the dynamics of artificial one- and two-dimensional Ising-like quantum antiferromagnets with different lattice geometries by using a Rydberg quantum simulator of up to 36 spins in which we dynamically tune the parameters of the Hamiltonian. We observe, in a region in parameter space, the onset of antiferromagnetic (AF) ordering, albeit with only finite-range correlations. We study systematically the influence of the ramp speeds on the correlations and their growth in time. We observe a delay in their buildup associated to the finite speed of propagation of correlations in a system with short-range interactions. We obtain a good agreement between experimental data and numerical simulations, taking into account experimental imperfections measured at the single-particle level. Finally, we develop an analytical model, based on a short-time expansion of the evolution operator, which captures the observed spatial structure of the correlations, and their buildup in time.

DOI: 10.1103/PhysRevX.8.021070

\section{INTRODUCTION}

The study of nonequilibrium dynamics is currently one of the most challenging areas of quantum many-body physics. In contrast to the equilibrium case, where statistical physics provides a general theoretical framework and where very powerful numerical methods are available, the out-of-equilibrium behavior of quantum matter presents a wide variety of phenomena and is extremely hard to simulate numerically, especially in dimensions $d>1$. When the parameters of a quantum many-body system are quenched abruptly or, more generally, ramped with a finite rate into a new quantum phase, correlations and entanglement build up and propagate over the system, which may, at long times, either thermalize or retain memory of its initial state when many-body localization occurs $[1,2]$. The speed at which the correlations propagate depends on the range of the interaction and is limited by the Lieb-Robinson bounds [3-8].

${ }^{*}$ V. L., S. de L., and M. S. contributed equally to this work.

Published by the American Physical Society under the terms of the Creative Commons Attribution 4.0 International license. Further distribution of this work must maintain attribution to the author(s) and the published article's title, journal citation, and DOI.
Subject Areas: Atomic and Molecular Physics,

Condensed Matter Physics, Magnetism,

Quantum Physics
An attractive way to study this physics has emerged in recent years and consists of using quantum simulators, i.e., well-controlled, artificial quantum systems that implement experimentally the Hamiltonian of interest [9]. Spin Hamiltonians that are used in condensed-matter physics to describe, e.g., quantum magnets are arguably the simplest quantum many-body systems that can be used to study nonequilibrium dynamics: Even though they involve distinguishable particles with only internal degrees of freedom, the interplay among interactions, geometry, and dimensionality provides a wealth of distinct quantum phases into which the system can be driven. In recent years, many experimental platforms for the quantum simulation of spin Hamiltonians have been developed using the tools of atomic physics. For example, equilibrium properties of synthetic quantum magnets have been studied using trapped ions $[10,11]$ or ultracold atoms in optical lattices [12-16], including, e.g., the observation of long-range antiferromagnetic order [17]. Many experiments using these platforms were also devoted to the study of nonequilibrium dynamics, including the investigation of the Lieb-Robinson bound [18-24].

Recently, a new platform, using arrays of individually resolved atoms excited to Rydberg states, has been shown to provide a versatile way to engineer synthetic quantum Ising magnets [25]. Pioneering experiments in quantum gas microscopes studied quenches [26] or slow sweeps [27] in a 
(a)
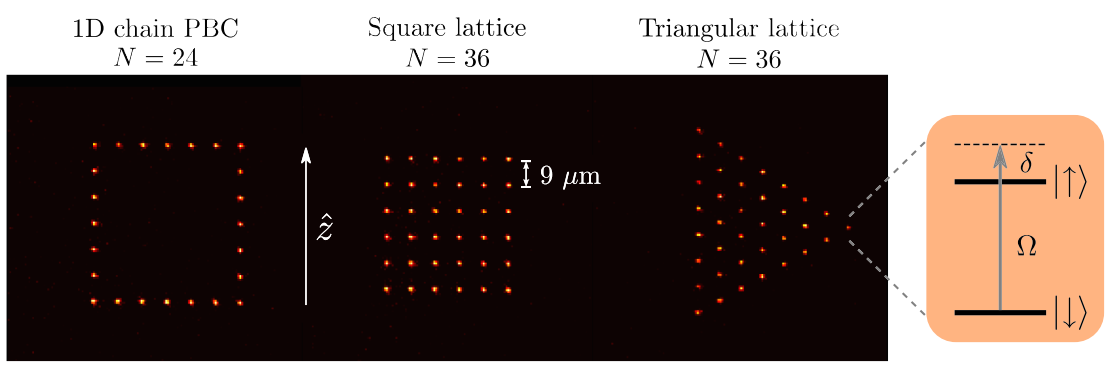

(b)

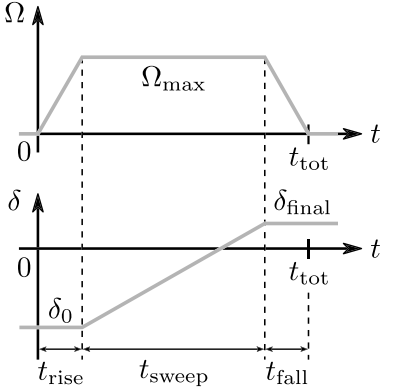

(c)
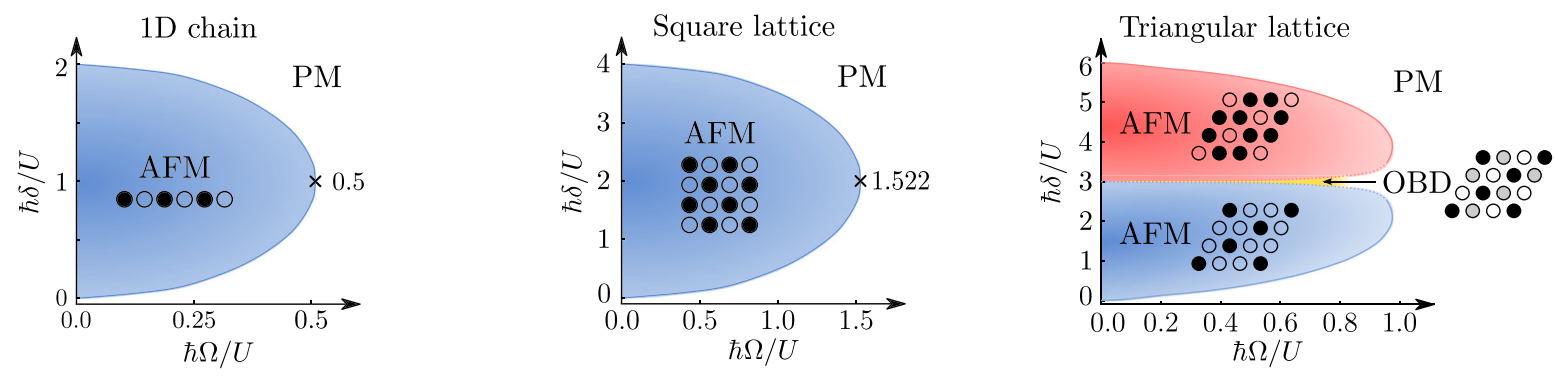

(d)
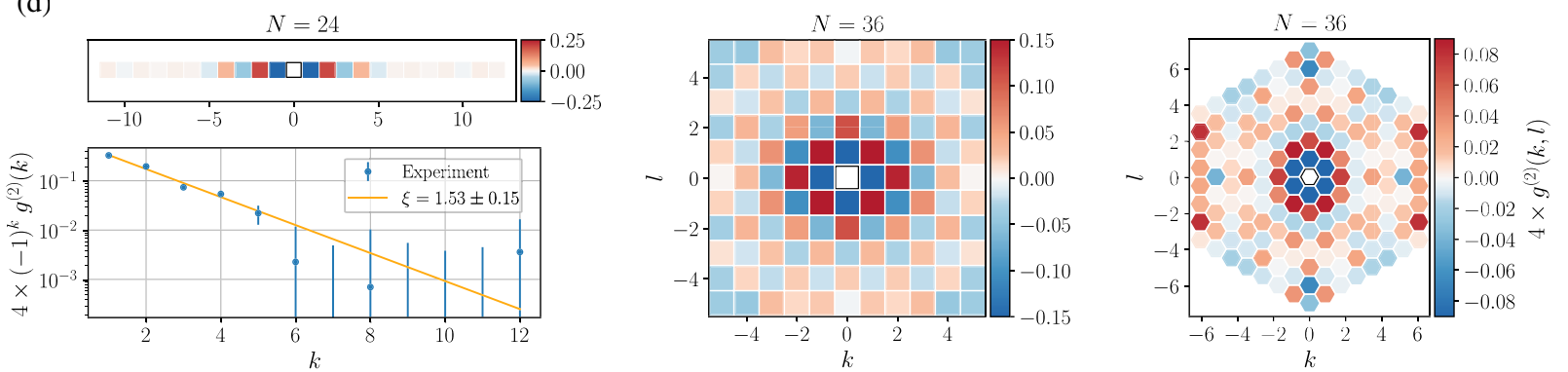

FIG. 1. Studying the AF Ising model on $1 \mathrm{~d}$ and $2 \mathrm{~d}$ systems. (a) Examples of single-shot fluorescence images of single-atom arrays used in our experiments: a 24-atom 1d chain with periodic boundary conditions, a $6 \times 6$ square array, and a 36-atom triangular array. Each atom is used to encode a spin-1/2, whose internal states $|\uparrow\rangle$ and $|\downarrow\rangle$ are coupled with Rabi frequency $\Omega$ and detuning $\delta$. (b) Time dependence of the Rabi frequency $\Omega(t)$ and detuning $\delta(t)$ used to probe the buildup of correlations. (c) Sketched ground-state phase diagrams of the Ising model in Eq. (1), in the nearest-neighbor interaction limit, for a $1 \mathrm{~d}$ chain, a $2 \mathrm{~d}$ square lattice, and a $2 \mathrm{~d}$ triangular lattice. In the figure, AFM stands for antiferromagnetic, PM for paramagnetic, and OBD for order by disorder. (d) Typical experimental correlation functions obtained for these geometries (see text). For the 1d chain, the correlation length $\xi=1.5$ sites (bottom left panel).

regime where the blockade radius $R_{b}$, i.e., the distance over which interatomic interactions prevent the excitation of two atoms, was much larger than the lattice spacing $a$, rendering the underlying lattice hardly relevant. In this case, the observed correlations are liquidlike, and observing the crystal-like ground state of the system [28] would require exponentially long ramps [29]. More recently, experiments with arrays of optical tweezers allowed exploring the regime $R_{b} \gtrsim a$, studying nonequilibrium dynamics following quenches [30] or slow sweeps [31].

Here, we use a Rydberg-based platform emulating an Ising antiferromagnet to study the growth of correlations during ramps of the experimental parameters in $1 \mathrm{~d}$ and $2 \mathrm{~d}$ arrays of up to 36 single atoms with different geometries. We operate in the regime $R_{b} \simeq a$, where the interactions act to a good approximation only between nearest neighbors. We dynamically tune the parameters of the Hamiltonian and observe the buildup of antiferromagnetic order. We also observe the influence of the finite ramp speed on the extent of the correlations, and we follow the development in space and time of these correlations during a ramp. Numerical simulations of the dynamics of the system without any adjustable parameters are in very good agreement with the experimental data and show that single-particle dephasing arising from technical imperfections currently limits the range of the observed correlations. Finally, we observe a characteristic spatial structure in the correlations, which can be understood qualitatively by a short-time expansion of the evolution operator for both square and triangular lattices. This study is a benchmarking of a state-of-theart quantum simulator of spin models in nontrivial settings (two-dimensional geometries, including frustrated ones). It shows that, although single-particle dephasing is so far a limitation for the study of ground-state properties, it does not prevent the observation of interesting features in the dynamics of these systems, in particular concerning the propagation of correlations during dynamical tuning of the parameters. 
TABLE I. Experimental parameters used for the data presented in the main text.

\begin{tabular}{lcccccc}
\hline \hline Figures & $U / h(\mathrm{MHz})$ & $\Omega_{\max } /(2 \pi)(\mathrm{MHz})$ & $\delta_{\text {final }} /(2 \pi)(\mathrm{MHz})$ & $t_{\text {rise }}(\mu \mathrm{s})$ & $t_{\text {sweep }}(\mu \mathrm{s})$ & $t_{\text {fall }}(\mu \mathrm{s})$ \\
\hline 2 & 1.0 & 2.3 & {$[-2,6]$} & 0.25 & $\left\{\left(\delta_{\text {final }}-\delta_{0}\right) /[2 \pi \cdot 10(\mathrm{MHz})]\right\}$ & 0.5 \\
3 & 2.7 & 1.8 & 4.5 & 0.25 & {$[0.1,1.3]$} & 0.25 \\
4 & 2.7 & 1.8 & 4.5 & 0.25 & 0.44 & 0.25 \\
$5(\mathrm{a})$ & 2.7 & 1.8 & 4.5 & 0.25 & 0.44 & 0.25 \\
$5(\mathrm{~b})$ & 0.8 & 0.6 & 1.6 & 0.25 & 5.5 & 0.25 \\
\hline \hline
\end{tabular}

The paper is organized as follows. In Sec. II, we describe the experimental platform. After recalling the phase diagram for the different array geometries (Sec. III), we explore its impact on the buildup of correlations on the square array (Sec. IV). In Sec. VA, we study the influence of ramp speeds on the correlations. In Sec. V B, we observe a delay in the buildup of the spin-spin correlations, a feature linked to their finite speed of propagation. Finally, in Sec. VC, we analyze the $2 \mathrm{~d}$ spatial structure of the AF correlations on the square and triangular geometries and show that it is qualitatively captured by an analytical model based on short-time expansion.

\section{EXPERIMENTAL PLATFORM}

Our experimental platform (see Appendix A) is based on user-defined two-dimensional arrays of optical tweezers, each containing a single ${ }^{87} \mathrm{Rb}$ atom [30]. Here, we use the arrays shown in Fig. 1(a) containing up to $N=36$ atoms: a $1 \mathrm{~d}$ chain with periodic boundary conditions (PBC), a square lattice, and a triangular lattice. We achieve full loading of the arrays using our atom-by-atom assembler [32]. The atoms are prepared in the ground state $|\downarrow\rangle=$ $\left|5 S_{1 / 2}, F=2, m_{F}=2\right\rangle$ by optical pumping and then coupled coherently to the Rydberg state $|\uparrow\rangle=\mid 64 D_{3 / 2}$, $\left.m_{j}=3 / 2\right\rangle$, with a two-photon transition of Rabi frequency $\Omega$ and a detuning $\delta$, while the traps are switched off. The system is described by the Hamiltonian

$$
H=\sum_{i}\left(\frac{\hbar \Omega(t)}{2} \sigma_{i}^{x}-\hbar \delta(t) n_{i}\right)+\frac{1}{2} \sum_{i \neq j} U_{i j} n_{i} n_{j},
$$

where $n_{i}=|\uparrow\rangle\left\langle\left.\uparrow\right|_{i}\right.$ is the projector on the Rydberg state for atom $i$, and $\sigma^{x}=|\uparrow\rangle\langle\downarrow|+| \downarrow\rangle\langle\uparrow|$ is the $x$-Pauli matrix. The interaction term $U_{i j}$ arises from van der Waals interactions between the atoms and, thus, scales as $1 / r_{i j}^{6}$, with the distance $r_{i j}$ between atoms $i$ and $j$. This short-range character allows us to neglect interactions beyond nearest-neighbor (NN) atoms for this work [33], and we thus restrict Eq. (1) to NN terms only. For the $D$ states that we use, the van der Waals interaction is anisotropic [25,34], and the lattice spacings in the arrays are tuned such that the $\mathrm{NN}$ interactions anisotropy is below $10 \%$. We use typical values $\Omega_{\max } /(2 \pi) \sim 2 \mathrm{MHz}$ and $U / h \sim 1-3 \mathrm{MHz}$; see Table I. The driving parameters $\Omega$ and $\delta$ can be considered constant over the entire array (see Appendix A).

The system thus realizes an Ising-like model with a transverse field $\propto \Omega$ and a longitudinal field $\propto \delta$, which gives rise to antiferromagnetic (AF) order for $U>0$ (see below). We probe the system by using time-dependent ramps $\Omega(t)$ and $\delta(t)$, as shown in Fig. 1(b). The Rabi frequency $\Omega$ is switched on and off in $t_{\text {rise }}, t_{\text {fall }} \sim 250$ ns at a constant detuning $\delta$, and, in between, $\delta$ is ramped linearly from $\delta_{0} /(2 \pi)=-6 \mathrm{MHz}$ to $\delta_{\text {final }}$ during the time $t_{\text {sweep }}$. The total duration of the ramp is then $t_{\text {tot }}=t_{\text {rise }}+$ $t_{\text {sweep }}+t_{\text {fall }}$. After this, the trap array is switched on again. Atoms in $|\downarrow\rangle$ are observed by fluorescence, while those in $|\uparrow\rangle$ are lost from their trap. For a given set of parameters, the experiment is repeated a few hundred times to reconstruct quantities of interest such as the Rydberg density (equivalent to the magnetization), spin-spin correlation functions, or the sublattice density histogram. When $\delta_{\text {final }}$ lies in the AF region [see Fig. 1(c)], correlation functions show the emergence of short-range order [Fig. 1(d)] (see more details on the measurements in Sec. IV).

\section{THEORETICAL PHASE DIAGRAMS AND STATE PREPARATION CONSIDERATIONS}

As one of the stated goals of quantum simulations is the experimental exploration of phase diagrams of complex quantum many-body systems, we present an overview of the current understanding of the ground-state phase diagrams of the Hamiltonian under study. The calculated ground-state phase diagrams for the nearest-neighbor Ising model are shown in Fig. 1(c) for the three geometries considered in this paper. On the $1 \mathrm{~d}$ chain, the phase diagram is well known and features an AF phase and a paramagnetic phase delimited by a second-order quantum phase transition line of the $(1+1) d$ Ising universality class [35]. For $(\hbar \delta / U)_{\mathrm{TFI}}=z / 2$, with the coordination number $z=2$, the Hamiltonian corresponds exactly to the analytically solvable transverse field Ising (TFI) model without a longitudinal field, where in $1 \mathrm{~d}$ the critical point $(\hbar \Omega / U)_{c}=1 / 2$ is known analytically.

The phase diagram for the NN Hamiltonian on the square lattice $(z=4)$ is qualitatively similar to the phase diagram on the chain, with again an AF phase and a paramagnetic phase delimited by a second-order quantum phase transition line in the $(2+1) \mathrm{d}$ Ising universality class. For the transverse field Ising line $(\hbar \delta / U)_{\mathrm{TFI}}$, the critical point is 
known to high precision from Monte Carlo simulations, $(\hbar \Omega / U)_{c}=1.52219(1)$ [36]. Finally, on the triangular lattice $(z=6)$, the NN Hamiltonian features a much richer phase diagram. A Rydberg crystal with filling fraction $1 / 3$ (at $\Omega=0$ ), where one of the three triangular sublattices is occupied by Rydberg states and the atoms in the other sublattices remain in the ground state, appears in a region within $0<\hbar \delta / U<z / 2$. The conjugate crystals obtained by flipping all the spins (filling fraction $2 / 3$ at $\Omega=0$ ) lie in the region within $z / 2<\hbar \delta / U<z$. The most interesting feature occurs when $\hbar \delta / U=z / 2$, where an "order by disorder" process occurs [37]. When including the open boundary conditions for the square and triangular $N=36$ arrays studied here, the "phase diagrams" present the same phases with some modifications (see Appendix B).

In the experiments reported here, we initialize the system in the product state with all atoms in their ground state $|\downarrow\rangle$. Since the system is not at equilibrium with a thermal bath, we cannot simply cool to the ground state. Therefore, we use an experimental protocol involving sweeps of $\Omega(t)$ and $\delta(t)$ in order to characterize the ground states. Ideally, we would use adiabatic state preparation to reach the targeted ground state and would so be able to explore the phase diagrams of the highly nontrivial Hamiltonian in Eq. (1) on diverse lattices. In order for this approach to succeed, the duration of the sweep, which scales as the inverse of the square of the energy gap $\Delta$ above the instantaneous ground states [38], should be smaller than the coherence time of the system. In the $1 \mathrm{~d}$ chain and the $2 \mathrm{~d}$ square lattice, the minimal gap at the quantum phase transition scales as the inverse of the linear size of the system: $\Delta \sim 1 / N$ in $1 \mathrm{~d}$ and $\Delta \sim 1 / L=1 / \sqrt{N}$ in $2 \mathrm{~d}[35,39]$. The gap also depends on the excitation velocity at the quantum critical point, which can vary substantially along the quantum phase transition lines. For the triangular lattice, we expect a first-order quantum phase transition between the paramagnet to either the $1 / 3$ or $2 / 3$ filling Rydberg crystals, resulting in a minimal gap exponentially small in $N$ [40]. Because of these scalings, the gaps are small for the number of atoms used here. As a consequence, adiabatic state preparation would require long pulses. In the absence of any imperfections such as dephasing, pulse durations $t_{\text {tot }}$ of a few $\mu$ s would allow us to reach strong correlations extending over the entire system. While such durations are experimentally accessible, state-of-the-art platforms $[27,30,31]$ show significant dephasing over these timescales. For this reason, we approach here the question of state preparation from the opposite side and investigate the maximal strength of correlations that can be built, as well as their spatial structure, in a fixed amount of time. Answering these questions also informs us about the minimal time required to build up highly correlated states starting from a product state [4], a notion which is closely related to "quantum speed limits" in an optimal control context $[41,42]$.

\section{EXPLORING THE SQUARE LATTICE PHASE DIAGRAM}

In the first set of experiments, we explore the influence of the $\Omega=0$ "phase diagram" [see Fig. 1(c)] on the buildup of correlations on an $L \times L$ square array with $L=4$ and $L=6$ after relatively short ramps. In order to do so, we use the ramps shown in Fig. 1(b), with $\hbar \Omega_{\max } / U=2.3$. From the analysis of the final fluorescence images, we reconstruct the Rydberg density $n=\sum_{i}\left\langle n_{i}\right\rangle / N$, and the connected spin-spin correlation function

$$
g^{(2)}(k, l)=\frac{1}{N_{k, l}} \sum_{(i, j)}\left[\left\langle n_{i} n_{j}\right\rangle-\left\langle n_{i}\right\rangle\left\langle n_{j}\right\rangle\right],
$$

where the sum runs over atom pairs $(i, j)$ whose separation is $\boldsymbol{r}_{i}-\boldsymbol{r}_{j}=(k a, l a)$, and $N_{k, l}$ is the number of such atom pairs in the array [43]. For a perfect antiferromagnetic Néel ordering, $g^{(2)}$ takes the values $\pm 1 / 4$ for $|k|+|l|$ even and odd, respectively.

Figure 2(a) shows the spin-spin correlation function at the end of the ramp as a function of $x=\hbar \delta_{\text {final }} / U$. We observe strong AF correlations; i.e., the sign of $g^{(2)}(k, l)$ changes according to the parity of the Manhattan distance $|k|+|l|$, in the region $0<x<4$ where $\mathrm{AF}$ order is expected, while the correlations vanish outside of this region. The amplitude of the AF correlations decreases with distance, in a way that is well captured by an exponential decay with a correlation length $\xi$, defined by $g^{(2)}(k, l) \propto(-1)^{|k|+|l|} \exp [-(|k|+|l|) / \xi]$, of about 1.5 sites [see Figs. 1(d) and 3(b)]. Repeating the same experiment with a 1d chain yields the same correlation length (the particularity of the triangular lattice is discussed in Appendix F). Importantly, even though the correlation length is smaller than two sites for both the 1d chain and the square lattice, we are able to detect finite correlations with the expected sign structure for up to five Manhattan shells, i.e., almost over the whole array.

Another way to highlight AF correlations is to partition the array into the two Néel sublattices $A$ and $B$ and plot a two-dimensional histogram $P\left(n_{A}, n_{B}\right)$ with the $|\uparrow\rangle$ populations $n_{A}$ and $n_{B}$ of each sublattice as axes. For a perfect AF ordering, one would observe populations only in the two corners $\left(n_{A}=0, n_{B}=N / 2\right)$ and $\left(n_{A}=N / 2, n_{B}=0\right)$. The experimental results in Fig. 2(b) show that, for $x=2.5$ (central plot), the sublattice population histogram is substantially elongated along the antidiagonal $n_{B}=N / 2-n_{A}$, which is not observed for $x<0$ or $x>4$. For comparison, the dotted circles indicate the shape of the sublattice histograms that would be obtained for an uncorrelated random state with the same average Rydberg density: There, the elongation along the antidiagonal is absent. In Fig. 2(c), we show as a comparison simulated histograms for pure Hamiltonian evolution for a lattice with $L=4$, 

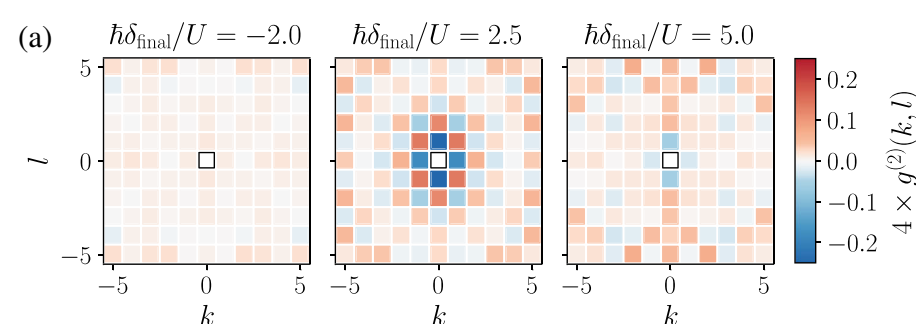

(b)
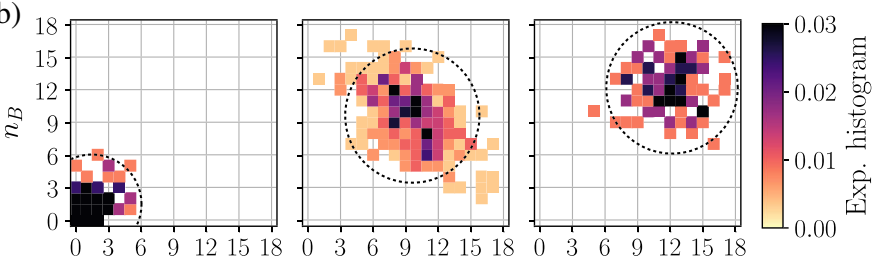

(c)
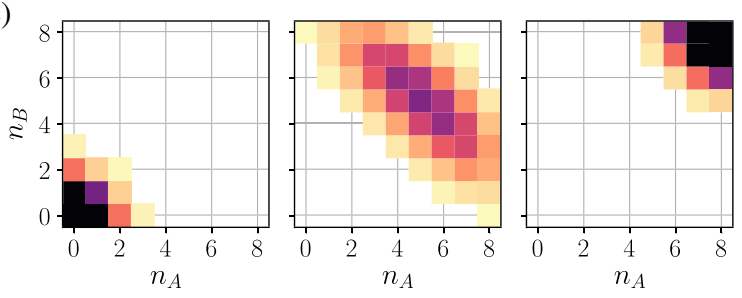

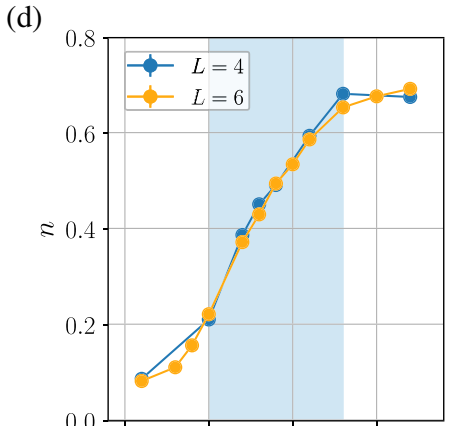

(e)

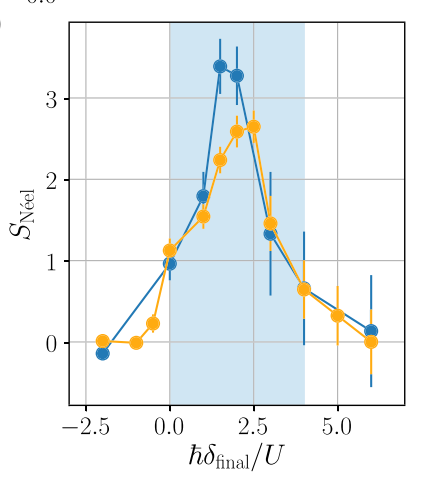

FIG. 2. Exploring the $\Omega=0$ line of the phase diagram for an $L \times L$ square lattice with $L=4$ and $L=6$. (a) Experimental spin-spin correlation function $g^{(2)}(k, l)$ for different values of the final detuning. (b) Corresponding experimental histograms of the populations of the two Néel sublattices $A$ and $B$, to be contrasted with those for uncorrelated random states with the same Rydberg density (the dotted circles indicate the positions where the random state histogram falls off to a value of $5 \times 10^{-4}$ ). (c) Simulated histograms (without dephasing) for $L=4$ as a comparison. (d) Average Rydberg density $n$, and (e) Néel structure factor $S_{\text {Néel }}$ as a function of the final detuning. The shaded areas highlight the region of the AF phase in the phase diagram for $\Omega=0$. In (d) and (e), the error bars (sometimes smaller than the symbol size) denote the standard error on the mean (s.e.m).

where we again see the typical elongation for the center panel induced by the short-range Néel ordering.

In Figs. 2(d) and 2(e), we attempt to locate the boundaries of the AF phase. Panel (d) shows the mean Rydberg density $n$. For the $\Omega=0$ ground state of Eq. (1) in the NN limit with periodic boundary conditions, it should rise in steps, from 0 for $x<0$, to $1 / 2$ for $0<x<4$, and to 1 for $x>4$ [44]. For open boundary conditions, additional steps are present in the $0<x<4$ region; see Appendix B. Experimentally, the curve $n(x)$ varies continuously due to the finite duration of the sweep, as also observed in Refs. [20,27]. This mean density is, therefore, not a good observable to differentiate the paramagnetic and $\mathrm{AF}$ regions. Instead, we introduce the Néel structure factor $S_{\text {Néel }}$ to detect antiferromagnetic correlations in the AF region of the phase diagram:

$$
S_{\text {Néel }}=4 \times \sum_{k, l \neq(0,0)}(-1)^{|k|+|l|} g^{(2)}(k, l)
$$

This quantity is an estimator for the correlation volume, i.e., the number of spins correlated antiferromagnetically with a given spin. In a situation with true long-range order, $S_{\text {Néel }}$ diverges linearly with the total "volume" $N$ of the system, while it stays almost constant for short-range ordered correlations when the system sizes are larger than the correlation length. As shown in Fig. 2(e), this quantity indicates the presence of substantial short-range AF correlations for $0<x<4$, which incidentally coincides with the antiferromagnetic region in the ground-state phase diagram.

The results presented so far demonstrate that antiferromagnetic correlations, although remaining short ranged, are strongest when the detuning at the end of the ramp is in the very same parameter range as the antiferromagnetic phase in the ground-state phase diagram $(0<x<4)$. We attribute this coincidence to the fact that the very different nature of the three phases (i.e., the Rydberg density zero state, the antiferromagnetic phase, and the completely Rydberg filled state) and the first order transitions between them occurring at $\Omega=0$ are discernible even to our relatively short ramps. In the next section, we will study how the correlations build up in time.

\section{EXPLORING THE TIME AND SPACE DEPENDENCE OF CORRELATIONS}

In the following, we first investigate in Sec. VA how $S_{\text {Néel }}$ depends on the ramp speed and find a duration $t_{\text {tot }}$ that maximizes its value. Then, with these settings, we study in 
(a)

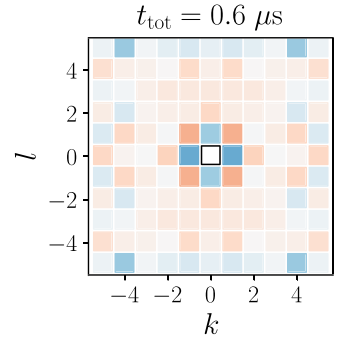

(b)

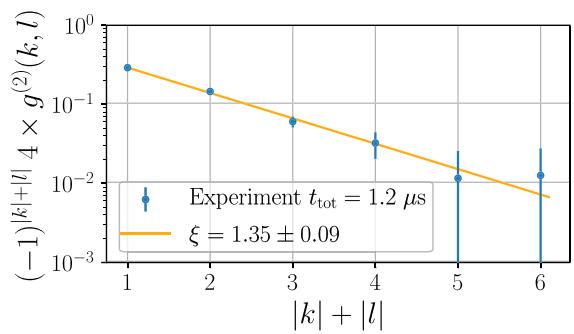

(d)

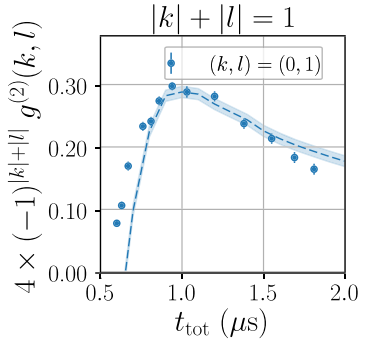

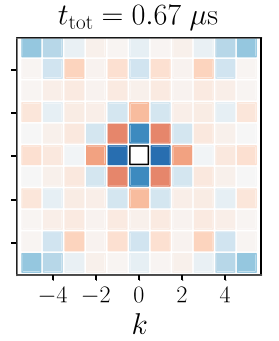
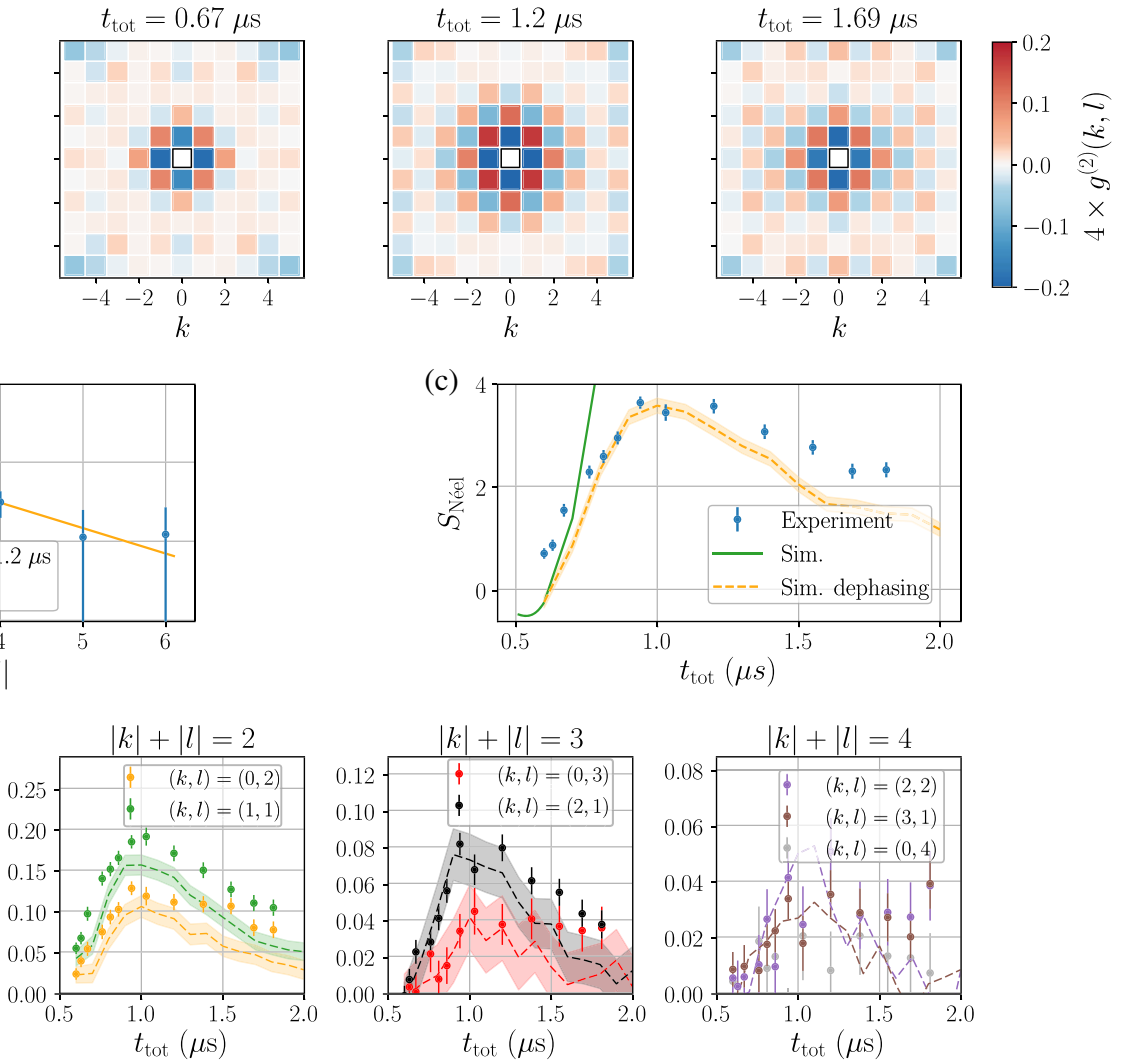

FIG. 3. Buildup and decay of correlations in a $2 \mathrm{~d}$ square lattice antiferromagnet for different ramp durations $t_{\text {tot }}$. (a) Experimental results for the $2 \mathrm{~d}$ correlation function $g^{(2)}(k, l)$ for four different values of $t_{\text {tot }}$, showing short-range AF ordering, with a pronounced sweep duration dependence. (b) The correlation function $g^{(2)}$ (averaged within a Manhattan shell $m=|k|+|l|$ ) decays exponentially with the Manhattan distance $m$, with a correlation length $\xi$ of about 1.4 lattice sites. (c) Néel structure factor $S_{\text {Néel }}$ as a function of $t_{\text {tot }}$. The green solid line shows the result of a numerical simulation using exact diagonalization for a $4 \times 4$ system without dephasing, while the yellow dashed line denotes a numerical simulation including dephasing (see Appendix C). (d) Dependence of the correlations on $t_{\text {tot }}$ for nearest, second-nearest, third-nearest, and fourth-nearest neighbors. One observes a dependence not only on the Manhattan distance $|k|+|l|$, but also on $k-l$ (see Sec. V C). The dashed lines represent a numerical simulation including dephasing (the shaded area corresponds to the s.e.m. obtained therein).

Sec. V B the temporal buildup of correlations during the optimized sweep and observe delays between the growth of correlations at increasing distances. Eventually, in Sec. V C, we analyze the spatial structure of these correlations and give a qualitative explanation of the observed patterns in terms of a short-time expansion.

\section{A. Correlations after ramps of varying durations}

We perform experiments on a 36-site square lattice using the sweep shown in Fig. 1(b) with fixed parameters $\hbar \delta_{\text {final }} / U=1.7, \hbar \Omega_{\max } / U=0.7<(\hbar \Omega / U)_{c}$. In contrast to the previous experiment, the quantum phase transition line is crossed while ramping the detuning $\delta$, and we vary the duration of the sweep $t_{\text {sweep }}$ (and, therefore, $t_{\text {tot }}$ ). The initial parameters are chosen such that the ground state at $t=0$ corresponds to $|\downarrow \downarrow \cdots\rangle$ (no Rydberg excitation) and the ground state at the final parameters is an AF [45].

Figure 3(a) shows two-dimensional plots of the experimental $g^{(2)}$ correlation functions for four different values of $t_{\text {tot }}$. For the shortest sweeps, the correlations remain weak. For intermediate durations $\left(t_{\text {tot }} \sim 1 \mu \mathrm{s}\right)$, strong correlations emerge, with a staggered structure extending over many Manhattan shells, as shown in Fig. 3(b). For even larger sweep durations, the correlation signal decreases with increasing sweep duration. In Fig. 3(c), we show the value of $S_{\text {Néel }}$ as a function of $t_{\text {tot }}$ : starting from small values for short ramps, it exhibits a broad maximum for $t_{\text {tot }} \sim 1 \mu \mathrm{s}$ and slowly decreases for longer ramp durations.

In order to gain a better understanding of the behavior described above, we compare the data with numerical simulations of the dynamics of the system governed by the Hamiltonian of Eq. (1) with the full van der Waals interactions for the sweeps used in the experiment [46]. We show in Fig. 3(c) the results of the simulation on a $4 \times 4$ square lattice. The green line corresponds to a unitary evolution that models well the experiment only for short sweep durations. The dashed yellow line includes the decoherence through a simple, empirical model assuming 

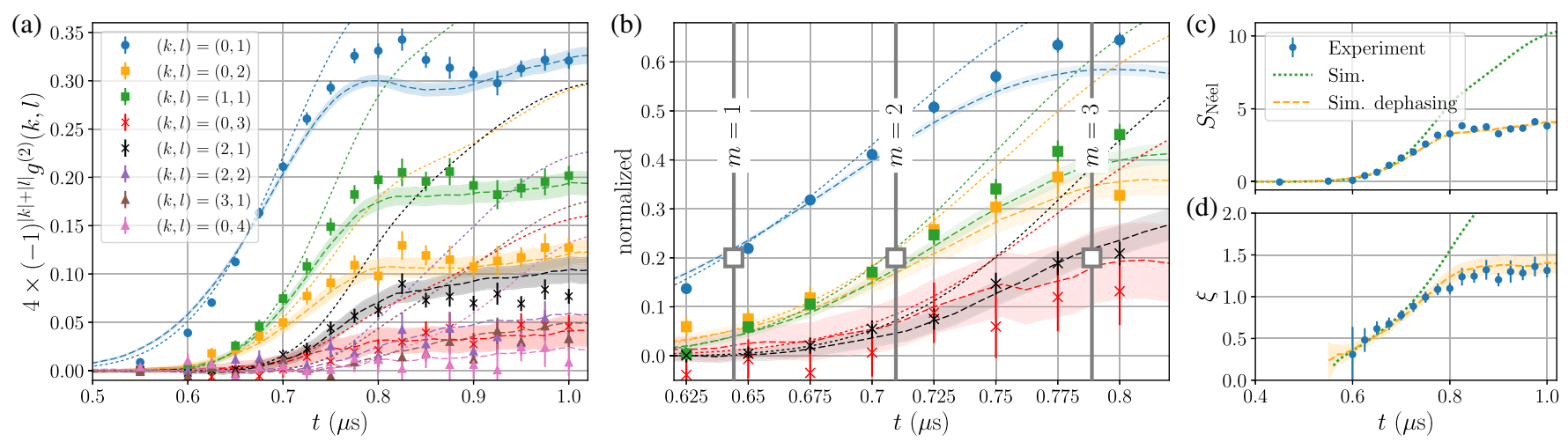

FIG. 4. Time evolution of a $6 \times 6$ square array along a ramp with $t_{\text {tot }}=1.0 \mu \mathrm{s}$. (a) Buildup of correlations along the ramp. (b) Observation of a time delay between the buildup of significant correlations in increasing Manhattan shells $m$. This is the manifestation of a finite propagation speed of correlations in their buildup between distant sites (see text). (c) Néel structure factor and (d) correlation length $\xi$. All figures: The dotted (dashed) lines correspond to the result of a numerical simulation for a $4 \times 4$ lattice without (with) dephasing.

a local (i.e., single-atom) dephasing, with a rate $\gamma \simeq$ $3.0 \mu \mathrm{s}^{-1}$ obtained from fits of single-atom Rabi oscillations (see Appendix C 2). We observe a remarkable agreement between this local dephasing model with no adjustable parameters and the experiment over a large range of $t_{\text {tot }}$. This indicates that the saturation and the decay of the correlations for longer sweeps are dominated by singleparticle decoherence, which makes it possible for numerical simulations on smaller systems to adequately model the observed correlation functions on the larger lattices realized in the experiment.

We now analyze the spin-spin correlations for different Manhattan distances $|k|+|l|$. In Fig. 3(d), we observe the buildup of correlations up to the fourth shell $|k|+|l|=4$, all of them being antiferromagnetically staggered, $g^{(2)}(k, l) \propto(-1)^{|k|+|l|}$. For short sweeps, the correlations sharply increase with increasing duration, saturate for longer sweeps, and decay, again due to decoherence. The simulation including dephasing (dashed lines) reproduces well this trend.

\section{B. Buildup of correlations along a ramp of fixed duration}

In a subsequent experiment, we analyze how the $\mathrm{AF}$ correlations build up in time, during the sweep that maximizes $S_{\text {Néel }}\left(t_{\text {tot }}=1.0 \mu \mathrm{s}\right)$ identified in the previous subsection. Stopping the dynamics after a variable time $0<t<t_{\text {tot }}$, Fig. 4 shows the time evolution of the correlations after entering the AF region identified in Fig. 2 at $\delta(t)>0(t>0.5 \mu \mathrm{s})$. We observe that most of the correlations build up for $0.6<t<0.8 \mu$ s and then freeze at larger times. When comparing again to the numerical simulation of the dynamics, including the single-particle dephasing, we obtain a remarkable agreement with the data.

Figure 4 also features a striking effect: We observe a delay in the buildup of correlations between the different
Manhattan shells $m=|k|+|l|$, highlighting the finite speed for the spread of correlations. To quantify this effect, we normalize the correlations for each distance such that the corresponding dephasing-free simulation reaches a maximum of 1 at large time [Fig. 4(b)]. Fixing an arbitrary threshold of 0.2 , we observe that the nearest neighbor shell $(m=1)$ reaches this threshold at $t \approx 0.64 \mu \mathrm{s}$, the second shell $(m=2)$ at $t \approx 0.71 \mu \mathrm{s}$, and, finally, the third shell ( $m=3$ ) at $t \approx 0.79 \mu$ s (see gray vertical lines). This delay is a manifestation of the finite propagation speed of correlations; such a finite propagation speed is theoretically predicted by Lieb-Robinson bounds [3,4]. Finite propagation speeds for correlations and other signals are well explored in the context of sudden quench protocols with constant postquench Hamiltonians [18,20,23,47,48], but their importance for state-preparation protocols (with a time-dependent Hamiltonian) is not equally well known (see Appendix D for a brief review on Lieb-Robinson bounds and effective propagation speeds adapted for the present context).

Figure 4(b) also reveals that when the correlations on the first shell reach the threshold, correlations for higher Manhattan distances are suppressed, but still detectable for $m=2$. This resembles the known fact that the correlations outside a Lieb-Robinson cone are finite, albeit exponentially suppressed (see also Appendix D). To recover this fact, we introduce a simple analytical approach based on a short-time expansion method (see Appendix E for details and specific results). We find analytical expressions in powers of the duration $T$ of the ramp for the connected $g^{(2)}$ correlation functions for different Manhattan distance $m$ valid in the limit $(U T / \hbar, \Omega T, \delta T \ll 1)$. We obtain nearest-neighbor correlations ( $m=1)$ of order $T^{6}$ and next-nearest-neighbor correlations $(m=2)$ of order $T^{10}$. More generally, the leading order of the expansion for two sites separated by a Manhattan distance $m$ appears at order $T^{2+4 m}$, thus suggesting that at a given (short) time the correlation decreases exponentially 

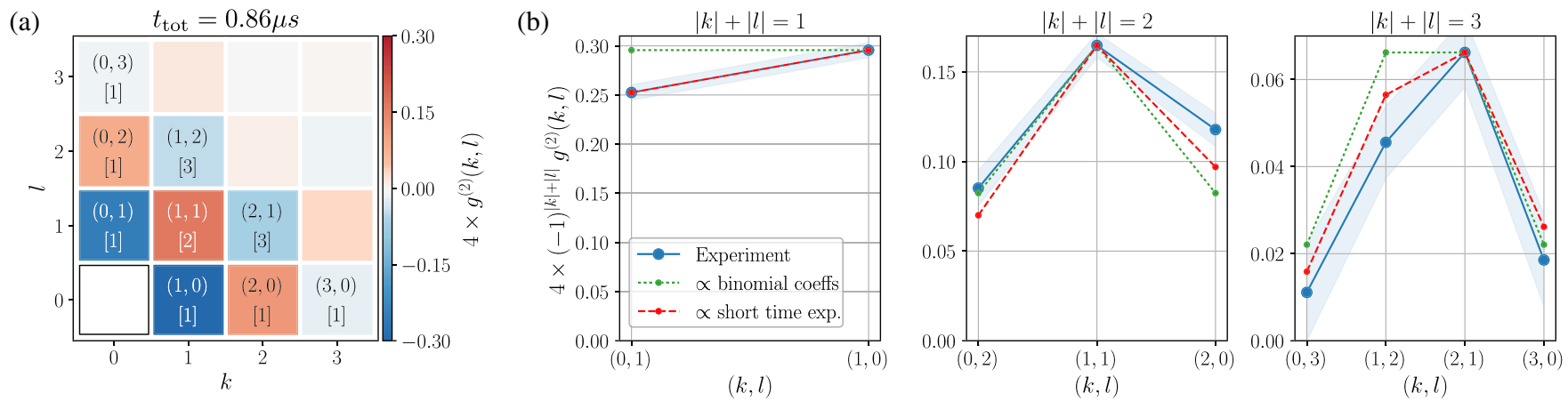

(c)

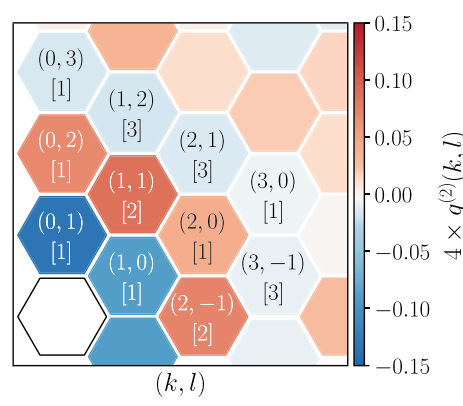

(d)

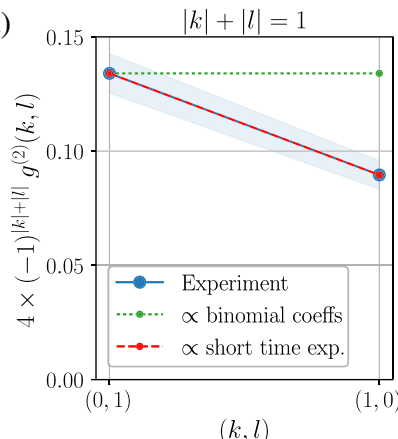

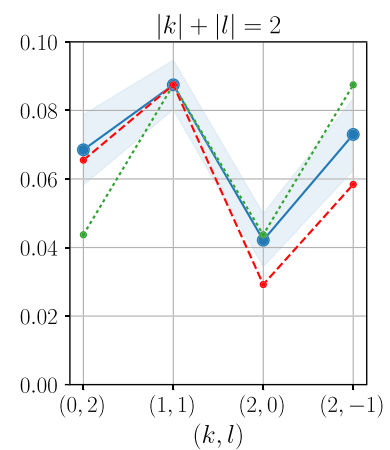

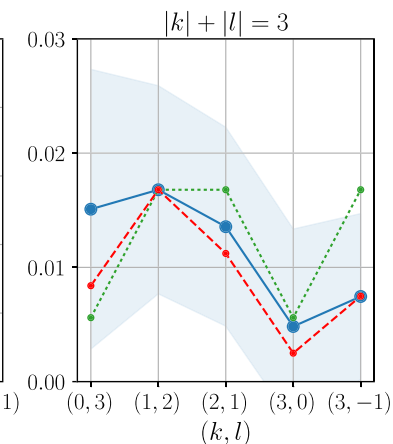

FIG. 5. Spatial structure of the spin-spin correlations on the 36-site square (a),(b) and on the 36-site triangular lattice (c),(d). Experimental data after a sweep is shown in (a) and (c), together with the values of $(k, l)$ for the first three shells $|k|+|l|$ [see Fig. 1 (d) for the full range]. The numbers in brackets $[c]$ give the number of linking paths contributing to the short-time expansion. (b) and (d) Dependence of the correlation on $(k, l)$ for several Manhattan distances with a qualitative comparison to binomial coefficients (green dotted lines) and leading-order short-time expansion (red dashed lines); see text for details. The shaded region corresponds to the s.e.m. of the experimental data.

with $m$ (see more details in Appendix E). This scaling explains qualitatively the observed time dependence in Figs. 4(a) and 4(b), even though, strictly speaking, the range of applicability of the short-time expansion is limited to times much shorter than those shown there (for which the correlations would still be extremely small, and thus, very challenging to measure experimentally). However, our numerical results without and with dephasing, as well as the experiment, show that the qualitative features of the shorttime expansion prevail for the considered nonperturbative times and the actual ramp shapes.

\section{Buildup of spatial structures on the square and triangular lattices}

We finally analyze the spatial structure of the correlations in more detail. Figures 3(d), 4(a), and 4(b) show that the correlations do not depend only on the Manhattan distance $m=|k|+|l|$, but also, for fixed $m$, on $k-l$ $[49,50]$. For instance, for second neighbors $(m=2)$, the correlations for $(k, l)=(0,2)$ or $(2,0)$ are about half of those along the diagonal $(k, l)=(1,1)$. This spatial structure, absent in a $1 \mathrm{~d}$ setting, has not, to our knowledge, been experimentally observed in $2 \mathrm{~d}$.

The observed spatial structures in the correlations within a given Manhattan shell $m$ can also be captured by the short-time expansion. The leading order coefficient of a given correlator $g^{(2)}(k, l)$ depends on the number of paths on the lattice linking the two sites as detailed in Appendix E 2. Figure 5 shows a comparison of the spatial structure in experimental data for both a square and a triangular lattice after a ramp of finite duration. In panels (a) and (c), the number of linking paths is given for $k \geq l \geq 0$ by the binomial coefficient $C_{k}^{m}$ for both lattices and is shown in brackets. In panels (b) and (d), we analyze the correlations on the square and triangular lattice. The green dotted lines show the $k-l$ dependence of $g^{(2)}(k, l)$, assumed to be given only by the binomial coefficients, normalized to the maximal correlation value for each subplot.

The precision of the experiment even allows us to observe a small asymmetry in each shell $m=$ const., because of a slight residual anisotropy of the interaction. An extension of the short-time expansion to the anisotropic case yields an analytical expression from which we extract the ratio $U_{z} / U_{w}$ of the nearest-neighbor interactions. We use this interaction anisotropy in the analytical expression to obtain the correlation for larger $|k|+|l|$. The results are shown as red dashed lines.

Interestingly, for triangular lattices, in contrast with the case of square arrays, the spatial structures of the correlations that one observes experimentally, and which are well reproduced by the short-time expansion, do not reflect 
directly those one would obtain in the ground state (see Appendix F). This specific behavior may be a dynamical signature of the frustrated character of the triangular geometry: In order for a system to explore its frustrated nature, large enough times are needed, such that the dynamics is able to explore the frustrated loops (i.e., triangles). In a perturbative expansion, like the short-time expansion, this means that large enough times are necessary such that the higher order contributions containing these loops are able to overcome the leading-order contributions, which, we have shown, are of a Manhattan-like structure. A detailed discussion is, however, beyond the scope of the present work.

\section{CONCLUSION AND OUTLOOK}

In conclusion, we have used a Rydberg-based platform to study antiferromagnetic correlations in a synthetic Ising magnet with different geometries. Using dynamical variations of the parameters, we explored the phase diagram and prepared arrays exhibiting antiferromagnetic order with pronounced Manhattan structures. We also studied the growth of the correlations during the sweeps of the parameters. We observed delays in the buildup of correlations between sites at different distances, a feature linked to the Lieb-Robinson bounds for the propagation of correlations in a system with nearest-neighbor interactions. We were able to understand the spatial structure of the correlations after short to intermediate evolution times using an analytical short-time expansion of the evolution operator. Finally, we obtained remarkable agreement between the data on the dynamics of the correlations and numerical simulations using a local (i.e., singleparticle) dephasing model.

In the future, the time over which coherent simulation can be performed in such artificial quantum magnets could be extended by a better understanding of the dephasing mechanisms at play in the coherent excitation of Rydberg states [51]. We will then be able to explore geometric frustration on triangular or Kagome lattices [52,53]. Alternatively, it could be interesting to explore the limit of strong dephasing to study dissipative phase transitions (see, e.g., Ref. [54]). Another promising avenue is the study of magnets described by the XY model implemented using resonant dipole-dipole interactions [55-57]: There, the coherent drive is obtained by using microwaves, and much lower dephasing rates are expected; moreover, the interaction decays slowly, as $1 / r^{3}$, and long-range effects should be more prominent [58].

\section{ACKNOWLEDGMENTS}

This work benefited from financial support by the EU [H2020 FET-PROACT Project RySQ], by the "Investissements d'Avenir" LabEx PALM (ANR-10LABX-0039-PALM, projects QUANTICA and XYLOS) and by the Région Île-de-France in the framework of DIM Nano-K. M. S., L. P. H., and A. M. L. acknowledge support by the Austrian Science Fund for project SFB FoQus (F-4018). The computational results presented have been achieved in part using the Vienna Scientific Cluster (VSC). This work was supported by the Austrian Ministry of Science BMWF as part of the UniInfrastrukturprogramm of the Focal Point Scientific Computing at the University of Innsbruck.

Note added.-Recently, similar antiferromagnetic correlations in $2 \mathrm{~d}$ have been observed at Princeton University by Elmer Guardado-Sanchez et al. [59], using a system of Li Rydberg atoms in a quantum gas microscope.

\section{APPENDIX A: EXPERIMENTAL DETAILS}

Here, we give more details about our experimental setup and on the mapping of the Rydberg system onto an Ising antiferromagnet.

We use a two-photon excitation scheme to excite the atoms to the Rydberg state. The two lasers at 795 and $475 \mathrm{~nm}$, with corresponding Rabi frequencies $\Omega_{r}$ and $\Omega_{b}$, and a detuning $\Delta \simeq 2 \pi \times 740 \mathrm{MHz}$ from the intermediate $\left|5 P_{1 / 2}, F=2\right\rangle$ state result in an effective Rabi frequency $\Omega=\Omega_{r} \Omega_{b} /(2 \Delta)$ for the coupling between $|\downarrow\rangle$ and $|\uparrow\rangle$, but also introduce lightshifts $\left(\Omega_{r}^{2}-\Omega_{b}^{2}\right) /(4 \Delta)$ that add up to the two-photon detuning $\delta$. In order to generate the ramps shown in Fig. 1(b), we thus compensate for these additional lightshifts. An AOM is used for changing dynamically the amplitude and frequency of the red beam. Because of the finite size of our excitation beams, the atoms do not all experience exactly the same $\delta$ and $\Omega$. For our arrays with a size of approximately $40 \mu \mathrm{m}$, the inhomogeneities of $\Omega$ are below $15 \%$, while the detuning does not differ from its value on the central atom by more than $150 \mathrm{kHz}$.

The detection of the state of each atom relies on the loss of atoms in the Rydberg state, which are not recaptured in the optical tweezers. This detection method is, thus, subject to small detection errors [51]. In particular, an atom actually in $|\downarrow\rangle$ has a small probability $\varepsilon$ to be lost and, thus, incorrectly inferred to be in $|\uparrow\rangle$. As in Ref. [30], we measure $\varepsilon$ in a calibration experiment, and then we include the effect of detection errors on all the theoretical curves.

An antiferromagnetic phase is expected when interactions between parallel spins are repulsive, corresponding to the case where $U>0$ in the Hamiltonian of Eq. (1). Figure 6(a) shows the energy levels for a $2 \times 2$ square matrix of atoms as a function of the detuning $\delta$, with $U>0$ and $\hbar \Omega \simeq 0.5 U$. The lowest energy configuration with two $|\uparrow\rangle$ spins is then an antiferromagnetic state for $0<\hbar \delta<2 U$. Then, starting from a detuning $\delta<0$, with all atoms in $|\downarrow\rangle$, and ramping it up to $\delta \approx U / \hbar$ (dashed arrow), the lowest energy state is evolving adiabatically to the antiferromagnetic state. In our experiment, the interactions between 
(a) $C_{6}>0$

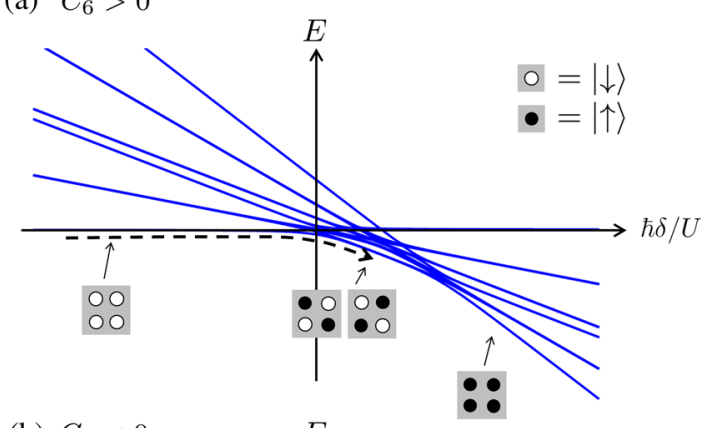

(b) $C_{6}<0$

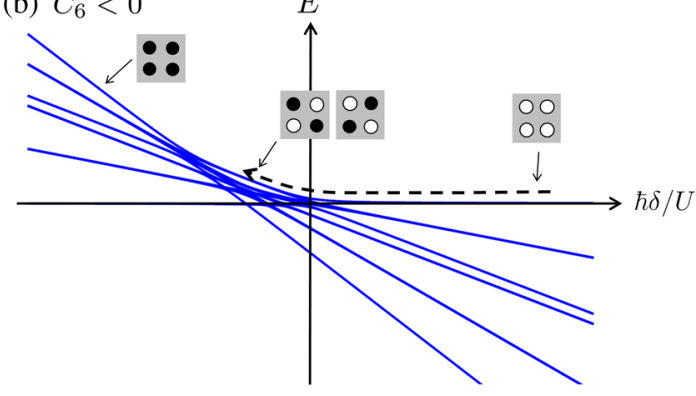

FIG. 6. Influence of the sign of $C_{6}$ on the many-body spectrum. Eigenenergies of a small $2 \times 2$ square system as a function of $\delta$ for $\hbar \Omega / U \simeq 0.5$. (a) For a repulsive interaction $C_{6}>0$, the ground state of $H$ is antiferromagnetic for $0<\hbar \delta<2 U$. It can be reached from $|\downarrow\rangle \otimes 4$ by adiabatically following the ground state starting from negative values of $\delta$ (trajectory in dashed arrows). (b) For an attractive interaction $C_{6}<0$, the antiferromagnetic state is the most excited state, and the sign of the detuning needed to reach it from $|\downarrow\rangle^{\otimes 4}$ is reversed.

$|\uparrow\rangle=\left|64 D_{3 / 2}, m_{j}=3 / 2\right\rangle$ spin states are in fact attractive $(U<0)$. Consequently, the antiferromagnetic state is never the lowest energy configuration whatever the value of $\delta$; see Fig. 6(b). Nevertheless, starting from a detuning $\delta>0$ and ramping it down to $\delta \approx U / \hbar$, the system evolves from the initial state to an antiferromagnetic state while following the highest-energy level. In this case, preparing the antiferromagnetic state actually means obtaining the ground state of $-H$ and, hence, the change of sign of the detunings needed to reach the correlated phase. For our parameters, the van der Waals interaction is not only attractive, but also slightly anisotropic $[25,34,60]$, being about three times as small in the horizontal direction as in the vertical one (along $\hat{z}$ ). We compensated for this difference by slightly distorting the arrays along $\hat{z}$ by a factor of approximately $3^{1 / 6}$.

\section{APPENDIX B: FINITE-SIZE EFFECTS}

The two-dimensional arrays used here present open boundary conditions $(\mathrm{OBC})$. The lattice sites along the boundary have fewer neighbors than the sites in the bulk and, therefore, experience fewer interactions. This modifies the "phase diagram" with respect to the bulk phase diagrams presented in Fig. 1. In Fig. 7, we show the classical (i.e., $\Omega=0$ ) ground-state configurations for the
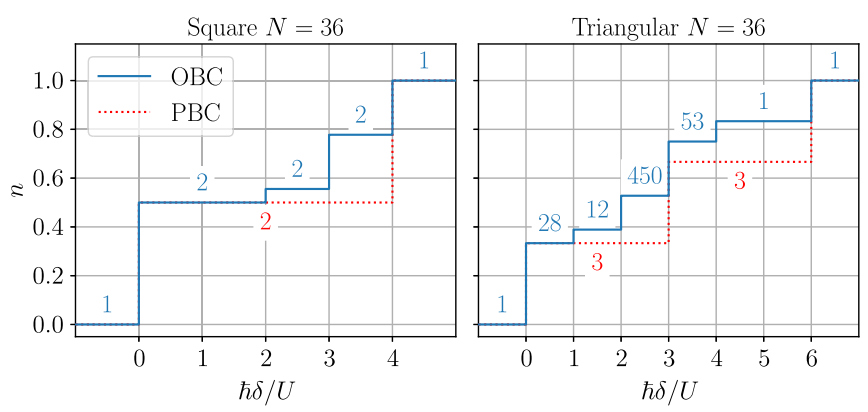

FIG. 7. Classical $(\Omega=0)$ phase diagrams for arrays with open boundary conditions, with Rydberg density $n$ as a function of $x=\hbar \delta / U$ for the $N=36$ square and triangular arrays used in the experiment. Blue lines correspond to open boundary conditions, while red dashed lines correspond to periodic boundary conditions (bulk phase diagram). The numbers indicate the degeneracy of the classical configurations for the distinct plateaus.

$N=36$ square and triangular arrays used in the experiment and compare them to the bulk phase diagrams. The sites at the boundary are more easily excited to the Rydberg state when $\delta$ increases, leading to a larger number of density plateaus. We have checked that, for the parameters used in this paper, the $\mathrm{OBC}$ and bulk phases feature the same typical short-range correlations.

The Néel structure factor $S_{\text {Néel }}$ shows almost no finite-size effects for short-range ordered systems as long as the system sizes are larger than the correlation length. For finite arrays, the number of pairs of sites with larger distances $|k|+|l|$ is strongly reduced, leading to larger errors for the connected correlations $g^{(2)}(k, l)$ due to reduced statistics. In order to keep reasonable values and errors for the Néel structure factor, we have restricted the summation to $|k|+|l| \leq 4$ in the experimental evaluation of $S_{\text {Néel }}$.

\section{APPENDIX C: NUMERICAL METHODS}

We perform numerical simulations of the time evolution on medium-size lattices for both Hamiltonian systems without any decoherence and the same systems with additional dephasing terms in the framework of a master equation in Lindblad form. In both cases, we construct the complete many-body Hilbert space of the system on the finite lattice and do not perform any truncation to calculate the time evolution. Therefore, during the time evolution, the system can explore the entire Hilbert space and all phases in the phase diagram can potentially be obtained.

\section{Unitary time evolution}

To simulate the unitary time evolution of a timedependent Hamiltonian $H(t)$, we approximate $H(t)$ by a piecewise constant Hamiltonian $H_{\text {approx }}(t)$ on $n_{\text {steps }}$ intervals of length $\Delta t$, such that 


$$
H_{\text {approx }}(t)=\frac{H(n \Delta t)+H((n+1) \Delta t)}{2},
$$

for $n \Delta t \leq t<(n+1) \Delta t$. We then compute the evolution operator within each interval with a Krylov-type matrix exponential approach [61] and use the evolved state of the previous interval as the starting state. We choose $n_{\text {steps }}$ such that the results do not differ from a simulation with $n_{\text {steps }} / 2$ intervals within a demanded accuracy. In most of the presented simulations, $n_{\text {steps }}=200$.

We are able to calculate the unitary time evolution of system sizes of up to about 30 lattice sites with a reasonable amount of resources. In the present study, most of the numerical results have been obtained on $4 \times 4$ and $5 \times 5$ square lattices. Because of the relatively short correlation lengths observed in the experiments, the numerical results on the smaller systems can nevertheless be used to model the observed correlation functions on the larger, experimentally realizable lattices.

\section{Time evolution in the presence of dephasing}

The experimental system features several sources of imperfections [51], and the description of its evolution as a pure Hamiltonian is not exact. In particular, phase noise of the excitation lasers and Doppler shifts lead to dephasing, already for a single particle. We thus perform simulations with a phenomenological local dephasing model with a rate $\gamma$ obtained by fitting experimental single-atom Rabi oscillations, as shown in Fig. 8(a). We obtain dephasing rates $\gamma \approx 3-4 \mu \mathrm{s}^{-1}$ for $\Omega /(2 \pi)=1.8 \mathrm{MHz}$ (for pure dephasing with a rate $\gamma$, the $1 / e$ damping time of the envelope of the Rabi oscillation is $4 / \gamma$ ). The time evolution of the density matrix $\rho(t)$ of the many-body system is then described by the following master equation in Lindblad form:

$$
\frac{d}{d t} \rho=-\frac{i}{\hbar}[H, \rho]+\mathcal{L}[\rho],
$$

with a Liouvillian

$$
\mathcal{L}[\rho]=\sum_{i} \frac{\gamma}{2}\left(2 n_{i} \rho n_{i}-n_{i} \rho-\rho n_{i}\right)
$$

Direct simulations of the master equation are only possible for small lattices as the memory demand for saving density matrices grows as $\mathcal{O}\left(4^{N}\right)$ for the considered Hilbert space on $N$ sites. Thus, we use a Monte Carlo wave function (MCWF) method [62-64], where only a single wave function has to be stored such that one can simulate system sizes of up to around 20 sites. The MCWF method evolves a starting state with an effective non-Hermitian Hamiltonian; at each time step, a quantum jump corresponding to the given decoherence operator collapses the evolved state with a given probability. Averaging over many such quantum trajectories-we use 1280 for the presented results-allows reconstructing the density matrix
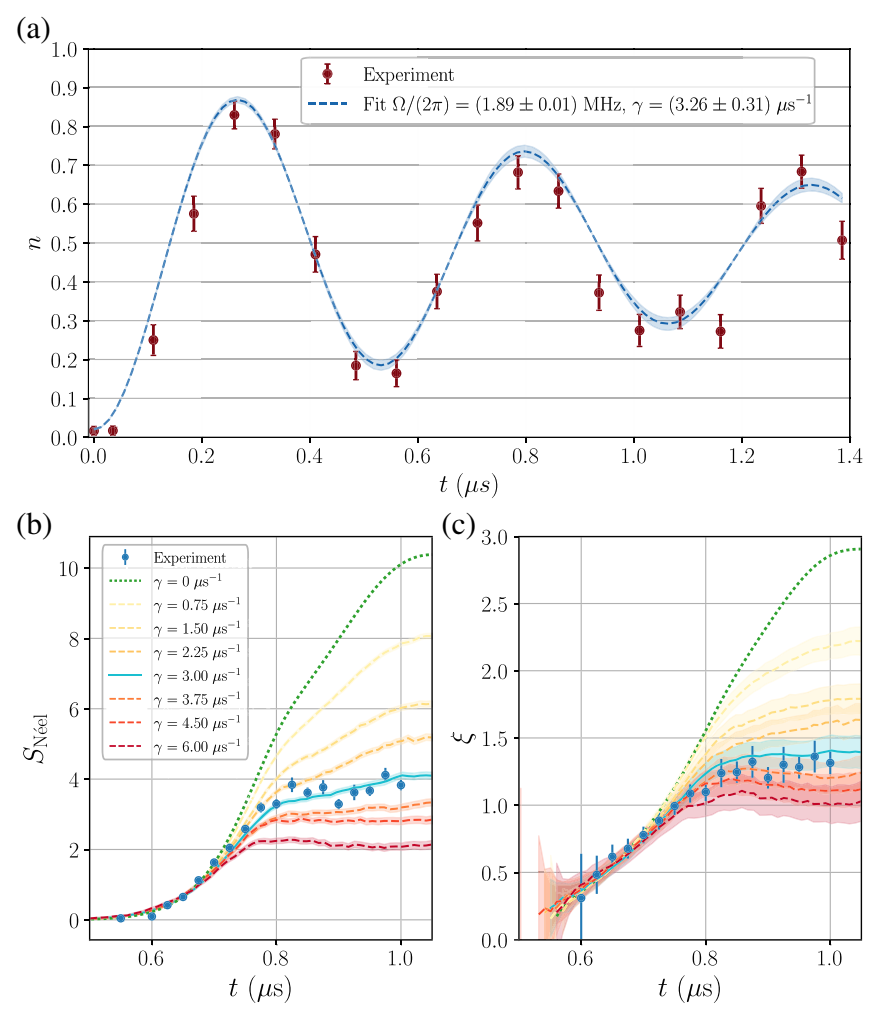

FIG. 8. (a) Single-atom Rabi oscillations, averaged over a typical square array (dark red symbols). We perform a fit (dashed line) to the analytical model including detection deficiency to obtain the dephasing rate $\gamma$ and the Rabi frequency $\Omega$. The value of $\gamma$ thus obtained is then used for the simulations of the experiments with $\Omega_{\max }=\Omega$ [cf. Figs. 3(c) and 3(d)]. (b),(c) Simulation of the many-body real-time evolution as in Figs. 4(c) and 4(d), with different values of the dephasing rate $\gamma$ for (b) the Néel structure factor and (c) the correlation length. The rate $\gamma=3 \mu \mathrm{s}^{-1}$ used in the main text as obtained in (a) is indicated by a solid line.

and, thus, computing any observable during the time evolution. In Figs. 8(c) and 8(d), we compare experimental data with simulations using different dephasing rates $\gamma$ and observe that the best agreement is indeed achieved by choosing $\gamma$ obtained from fitting the single-particle Rabi oscillations.

In practice, to perform simulations with dephasing, we use the Python toolbox "QuTiP" [65].

\section{APPENDIX D: LIEB-ROBINSON BOUNDS}

In nonrelativistic quantum mechanics, one might believe that information propagation is instantaneous because there is no explicit speed of light limiting the propagation. However, in 1972, Lieb and Robinson [3] proved that, in an extended quantum many-body system with a finitedimensional local Hilbert space and sufficiently local interactions, there is nevertheless a characteristic velocity 
emerging, which defines an approximate light cone for the propagation of information and implementing causality.

In Ref. [4], the Lieb-Robinson bound has been generalized to equal-time connected correlation functions of two operators (normalized to unity), acting on spatial regions $A$ and $B$ (of size $|A|$ and $|B|$ ), at a distance $d$ apart from each other, after some time evolution of duration $t$, and starting from an initial state with exponentially decaying correlations, with a correlation length $\chi$. Then, the connected equal-time correlation $g^{(2)}(d, t):=$ $\left\langle O_{A}(t) O_{B}(t)\right\rangle-\left\langle O_{A}(t)\right\rangle\left\langle O_{B}(t)\right\rangle$ is bounded as follows:

$$
\left|g^{(2)}(d, t)\right| \leq \bar{c}(|A|+|B|) \exp \left[-(d-2 v t) / \chi^{\prime}\right]
$$

with $\chi^{\prime}=\chi+2 \zeta$. The coefficients $\bar{c}, v$, and $\zeta$ depend on the Hamiltonian and the considered operators $O_{A}$ and $O_{B}$. The velocity $v$ is particularly important and is called the LiebRobinson velocity.

The importance of this result for the present study is that correlations at a distance $d$ are exponentially suppressed in $(d-2 v t) / \chi^{\prime}$ as long as the time $t<d /(2 v)$. After that time, the correlations are bounded by a number of $\mathcal{O}(1)$. This time dependence is visualized in Fig. 9.

We want to point out that the Lieb-Robinson theorem proves rigorous theoretical bounds on the speed with which phenomena can propagate in such systems; however, the actual dynamics does not necessarily saturate these bounds. One reason might be that the rigorous velocity bound is not tight, but the dynamics can be accurately modeled with a smaller velocity (as seems to be the case here; see below). More generally, however, the Lieb-Robinson bounds do not imply that signals have to spread ballistically, obeying a linear light cone. In most nonintegrable, ergodic systems, it is actually expected that signals propagate more slowly and show diffusive dynamics, thereby satisfying the LiebRobinson bounds broadly.

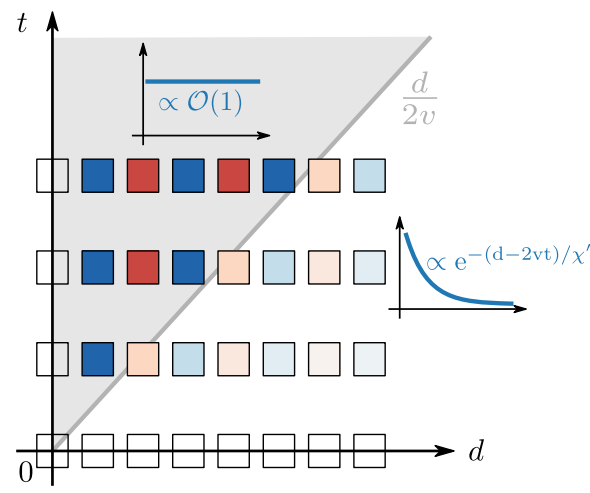

FIG. 9. The Lieb-Robinson bounds. "Significant" correlations spread along a light cone $d=2 v t$. Along and inside the light cone (grey shaded area), correlations of order $\mathcal{O}(1)$ can develop. Outside the light cone, $d>2 v t$ correlations are immediately built up for each $t>0$, but are exponentially suppressed in $d-2 v t$.
We have estimated the rigorous, maximal Lieb-Robinson velocity $[4,66]$ for our system in Eq. (1) restricted to nearestneighbor interactions and obtain $v=8 \mathrm{e}^{\max _{t}} F[U(t), \Omega(t)$, $\delta(t)]$, where we have absorbed the dependence on the coupling constants in a function $F$ and $\mathrm{e}=\exp (1)$; for the ramp used in Fig. 4, this evaluates to $v \approx 3.67 \times 2 \pi \times$ $8 \mathrm{e} a \mu \mathrm{s}^{-1}$ ( $a$ denotes the lattice spacing). The maximal velocity for the spreading of correlations $2 v$ is approximately 70 times faster than the observed time delay in Fig. 4(b), such that this experiment easily fulfills the bounds.

Obviously, the rigorous velocity bound is much larger than the velocity derived from the experimental time delay in the buildup of correlations. We try to address this discrepancy in the following. It has been observed in the past that, in some systems, the dynamically visible velocity-while bounded by the Lieb-Robinson velocity—can actually also depend on the initial state [67], a fact whose explanation requires a deeper knowledge of the dynamics, beyond the generic technology used to derive the Lieb-Robinson bounds.

In Ref. [68], the picture was introduced that quasiparticle excitations on top of a state are responsible for the lightcone-like spreading of correlations. The spreading of such quasiparticles-often computed based on effective models for a given physical situation-gives rise to a maximal spreading velocity, typically regarded as an effective LiebRobinson velocity $[18,69,70]$, although it often does not imply rigorous bounds. In order to obtain such effective velocities for our strongly interacting Hamiltonian, we apply a linear spin-wave theory approach: For a given set of Hamiltonian parameters, we compute the classical ground state and its semiclassical spin-wave excitation spectrum using standard linear spin-wave theory. From the soobtained dispersion relation $\epsilon(\mathbf{k})$, we compute the maximum magnon (group) velocity $v_{M}=(1 / \hbar) \max _{\mathbf{k}}\left|\nabla_{\mathbf{k}} \epsilon(\mathbf{k})\right|$. Figure 10 shows this instantaneous spin wave velocity along the ramp used in Fig. 4 (see also Table I). We observe that the maximum velocity is reached when the ramp crosses the quantum phase transition line between the paramagnetic and antiferromagnetic phase at $t \approx 0.5 \mu \mathrm{s}$; this maximal velocity

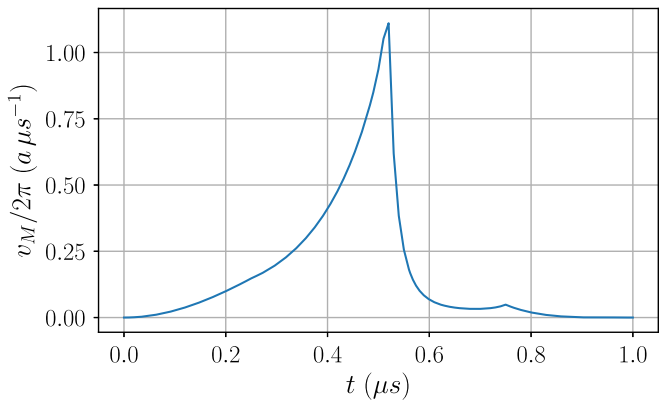

FIG. 10. Quasiparticle velocity along the ramp used in Fig. 4 obtained by linear spin-wave theory ( $a$ denotes the lattice spacing). The maximal velocity is reached when the ramp crosses the phase boundary at $t \approx 0.5 \mu \mathrm{s}$. 
$v_{\text {eff }} \approx 1.11 \times 2 \pi a \mu \mathrm{s}^{-1}$ can be regarded as an effective LiebRobinson velocity and is substantially smaller than the previously computed rigorous velocity. The correlation spreading rate $1 /\left(2 v_{\text {eff }}\right) \approx 70$ ns per lattice site is in good agreement with the time delays observed experimentally and numerically in Fig. 4.

\section{APPENDIX E: SHORT-TIME EXPANSION}

In this appendix, we provide more details on the shorttime expansion of the connected correlation function $g^{(2)}$.

\section{Theoretical description}

In order to simplify the notation, we use the nearestneighbor Hamiltonian $(\hbar=1)$ :

$$
H(t)=\sum_{\langle i, j\rangle} U_{i j} n_{i} n_{j}-\delta(t) \sum_{i} n_{i}+\Omega / 2 \sum_{i} \sigma_{i}^{x} .
$$

Here, we keep the possibility that nearest-neighbor interactions could be different from atom to atom. We consider simple ramp shapes characterized by a constant $\Omega$, a duration $T$, and a linear $\delta(t)$ dependence between $\delta(0)=$ $\delta_{0}$ and $\delta(T)=\delta_{\text {final }}$.

We are interested in the limit of very short duration $T$ and in particular how the correlations at various distances build up as we vary the duration $T$. Formally, we compute the full many-body propagator $\hat{U}(T)$, which solves the timedependent Schrödinger equation for the time-dependent Hamiltonian in Eq. (E1), allowing us to determine the many-body wave function at any time $T$ via $|\psi(T)\rangle=$ $\hat{U}(T)|\psi(0)\rangle$. We then express the connected correlation function as

$$
\begin{aligned}
g^{(2)}[(k, l)](T)= & \left\langle\psi(T)\left|n_{(0,0)} n_{(k, l)}\right| \psi(T)\right\rangle \\
& -\left\langle\psi(T)\left|n_{(0,0)}\right| \psi(T)\right\rangle\left\langle\psi(T)\left|n_{(k, l)}\right| \psi(T)\right\rangle .
\end{aligned}
$$

For the linear ramps considered here, a Magnus expansion [71] of the propagator is appropriate. We rely on the leading Magnus expansion term, which can be written as

$$
\hat{U}(T) \approx \exp \left[-i T H_{\mathrm{avg}}\right],
$$

with

$$
H_{\mathrm{avg}}=\frac{1}{T} \int_{0}^{T} H\left(t^{\prime}\right) d t^{\prime}
$$

For the ramp shape considered here, $H_{\text {avg }}$ is independent of $T$ and is of the same form as Eq. (E1), with $U_{i j}$ and $\Omega$ unchanged, while $\delta(t)$ is replaced by $\delta_{\text {avg }}=\left(\delta_{0}+\delta_{\text {final }}\right) / 2$.

We now calculate symbolic expressions for the power series in $T$ of the connected correlators in Eq. (E2), relying on the propagator in the leading Magnus expansion form and starting from an initial state with all sites in the atomic ground state. When $(U T / \hbar, \Omega T, \delta T \ll 1)$, we find the following expressions for the leading order in $T$ for a single path on a lattice, linking sites $(0,0)$ and $(k, l)$ (for the sake of simplicity, the results are only given for $U_{i j}=U$ ):

(i) nearest-neighbor correlation $|k|+|l|=1$

$$
g^{(2)}(T)=-\frac{1}{288}\left(U^{2}-3 U \delta_{\mathrm{avg}}\right) \Omega^{4} T^{6},
$$

(ii) next-nearest-neighbor correlation $|k|+|l|=2$

$$
g^{(2)}(T)=\frac{\Omega^{6} T^{10}}{2419200}\left(77 U^{4}-340 U^{3} \delta_{\mathrm{avg}}+375 U^{2} \delta_{\mathrm{avg}}^{2}\right),
$$

(iii) third-nearest-neighbor correlation $|k|+|l|=3$

$$
\begin{aligned}
g^{(2)}(T)= & -\frac{\Omega^{8} T^{14}}{26824089600}\left(4279 U^{6}-24766 U^{5} \delta_{\mathrm{avg}}\right. \\
& \left.+46725 U^{4} \delta_{\mathrm{avg}}^{2}-28350 U^{3} \delta_{\mathrm{avg}}^{3}\right) . \quad(\mathrm{E} 7)
\end{aligned}
$$

While the expressions look complicated, a common structure emerges: The leading $T$ dependence for a Manhattan distance $m=|k|+|l|$ is proportional to $T^{2+4 m}$. Neglecting the actual value of the coefficients for the moment in an admittedly crude approximation, these results suggest that correlations at a next shell are suppressed by a factor $T^{4}$ compared to the previous shell, suggesting an exponential spatial decay of the connected correlations at short times with $m$. These results also provide an insight as to why more distant shells require longer times to develop appreciable correlations: They require larger values of $T$ to overcome the initial suppression of the correlations resulting from the high powers in $T$.

Importantly, the neglected higher-order terms in the Magnus expansions do not alter the leading order in $T$ for the considered ramp shapes, but only affect the subleading $T$-coefficients. Finally, we note that, at very short times, correlations induced by the direct van der Waals tail of the interactions compete with the high-order nearest-neighbor considerations here. Although strictly speaking this leads to a breakdown of the exponential suppression of correlations beyond the light cone, the smallness of this effect prevents its observation in the present experiments.

\section{Lattice embedding}

The expressions derived above are lattice independent, as long as there is a chain of $m(m=|k|+|l|$ above) successive nearest-neighbor interactions linking the two considered sites [72]. These expressions can now be 

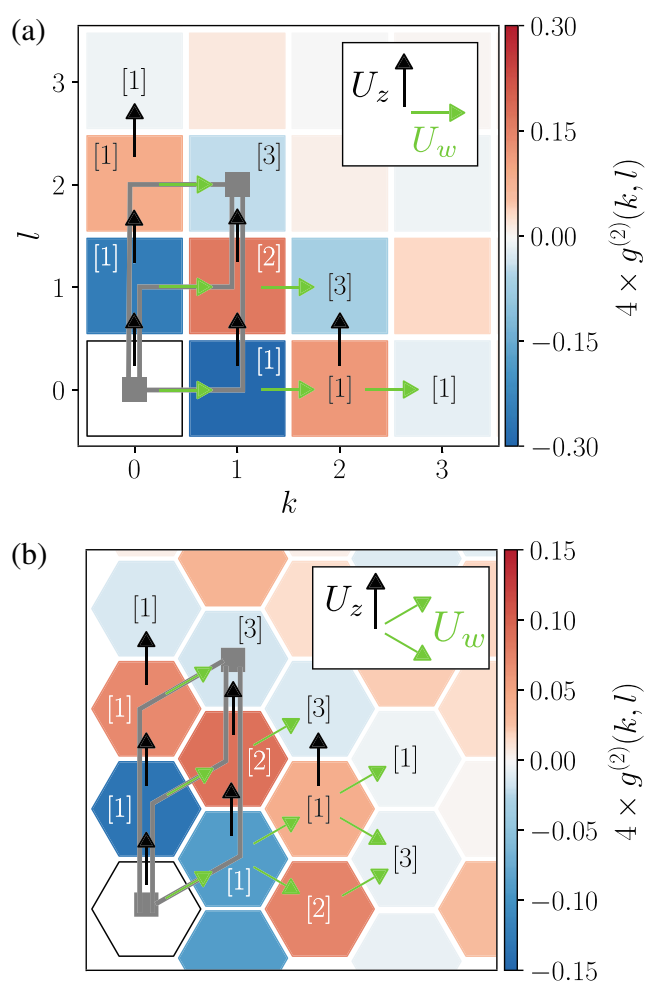

FIG. 11. Illustration of the different paths connecting two sites with a distance $(k, l)$ contribution to the leading-order short-time expansion on (a) the square lattice and (b) the triangular lattice. The number in brackets shows the binomial coefficient $C_{k}^{|k|+|l|}$ (assuming $k, l \geq 0$ ) corresponding to the number of distinct paths. The arrows show the potentially different couplings along paths contributing to the short-time expansion. The grey lines show the three distinct paths contributing to the correlation indicated by a grey square, as an example.

embedded in any lattice (e.g., cubic, Kagome, honeycomb, etc.), and the actual coefficients of the short-time series can be derived by determining the corresponding embedding coefficients. The embedding counting is illustrated in Fig. 11 for the square and the triangular lattice, yielding binomial coefficients multiplying the symbolic expressions for a single path derived above. Note that, for the case $U_{z} \neq U_{w}$, the coefficients of the single paths can already differ before taking the embedding factors into account.

\section{APPENDIX F: CORRELATIONS ON THE TRIANGULAR LATTICE: SHORT-TIME VERSUS GROUND-STATE CORRELATIONS}

The short-time expansion on the square lattice produces the same staggered correlation pattern as the one of the ground state of an AF ordered phase. There is, thus, a similarity between the sign structure of the correlations at short times and at very long times (i.e., the adiabatic limit).

On the triangular lattice, however, the correlations obtained in the experiment [see Fig. 12(a)], which are in good agreement with the short-time expansion as shown in
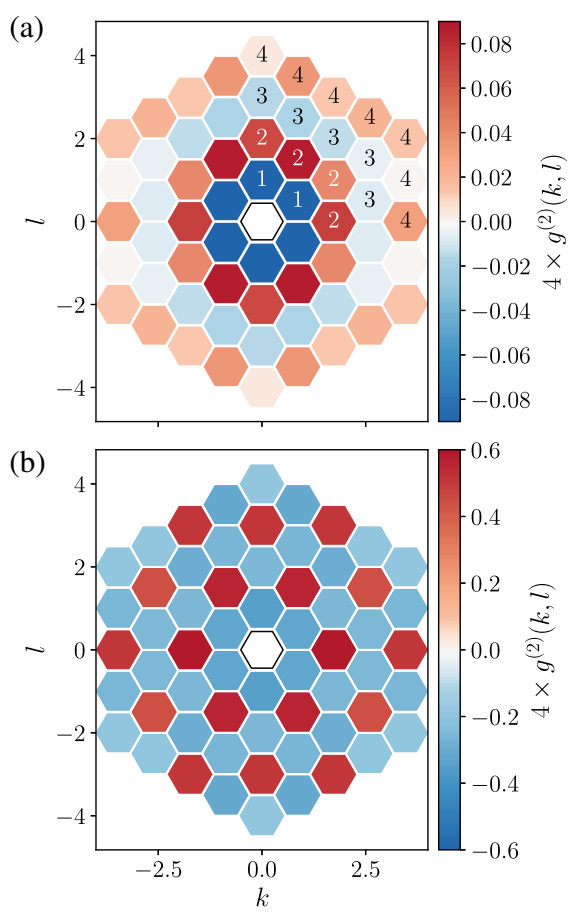

FIG. 12. Correlations on the $N=36$-site triangular array (only the first four Manhattan shells are shown). (a) Experimentally measured correlations after a sweep on the triangular array featuring a pronounced Manhattan shell structure $g^{(2)}(k, l) \propto$ $(-1)^{m}$. The numbers give the value of $m=|k|+|l|$. (b) Correlations of the equal superposition of the classical ground states for $\delta_{\text {final }}$ as reached at the end of the sweep used in (a). These correlations are in qualitative agreement with the correlations expected in the $1 / 3 \mathrm{AF}$ state in the right panel of Fig. 1(c).

Figs. 5(c) and 5(d), differ significantly from the ones expected for the AF ground state with density $1 / 3$ [Fig. 12(b)]. The origin of this discrepancy may be related to the frustrated character of the triangular geometry and will be the subject of future work.

[1] R. Nandkishore and D. A. Huse, Many-Body Localization and Thermalization in Quantum Statistical Mechanics, Annu. Rev. Condens. Matter Phys. 6, 15 (2015).

[2] E. Altman and R. Vosk, Universal Dynamics and Renormalization in Many-Body-Localized Systems, Annu. Rev. Condens. Matter Phys. 6, 383 (2015).

[3] E. H. Lieb and D. W. Robinson, The Finite Group Velocity of Quantum Spin Systems, Commun. Math. Phys. 28, 251 (1972).

[4] S. Bravyi, M. B. Hastings, and F. Verstraete, Lieb-Robinson Bounds and the Generation of Correlations and Topological Quantum Order, Phys. Rev. Lett. 97, 050401 (2006).

[5] J. Eisert, M. van den Worm, S. R. Manmana, and M. Kastner, Breakdown of Quasilocality in Long-Range Quantum Lattice Models, Phys. Rev. Lett. 111, 260401 (2013). 
[6] M. Foss-Feig, Z.-X. Gong, C. W. Clark, and A. V. Gorshkov, Nearly Linear Light Cones in Long-Range Interacting Quantum Systems, Phys. Rev. Lett. 114, 157201 (2015).

[7] I. G. White, B. Sundar, and K. R. A. Hazzard, Quantum Dynamics from a Numerical Linked Cluster Expansion, arXiv:1710.07696.

[8] L. Cevolani, G. Carleo, and L. Sanchez-Palencia, Spreading of Correlations in Exactly Solvable Quantum Models with Long-Range Interactions in Arbitrary Dimensions, New J. Phys. 18, 093002 (2016).

[9] I. M. Georgescu, S. Ashhab, and F. Nori, Quantum Simulation, Rev. Mod. Phys. 86, 153 (2014).

[10] K. Kim, M.-S. Chang, S. Korenblit, R. Islam, E. E. Edwards, J. K. Freericks, G.-D. Lin, L.-M. Duan, and C. Monroe, Quantum Simulation of Frustrated Ising Spins with Trapped Ions, Nature (London) 465, 590 (2010).

[11] P. Richerme, C. Senko, J. Smith, A. Lee, S. Korenblit, and C. Monroe, Experimental Performance of a Quantum Simulator: Optimizing Adiabatic Evolution and Identifying Many-Body Ground States, Phys. Rev. A 88, 012334 (2013).

[12] J. Simon, W. Bakr, R. Ma, M. Tai, P. Preiss, and M. Greiner, Quantum Simulation of Antiferromagnetic Spin Chains in an Optical Lattice, Nature (London) 472, 307 (2011).

[13] J. Struck, C. Ölschläger, R. Le Targat, P. Soltan-Panahi, A. Eckardt, M. Lewenstein, P. Windpassinger, and K. Sengstock, Quantum Simulation of Frustrated Classical Magnetism in Triangular Optical Lattices, Science 333, 996 (2011).

[14] R. Landig, L. Hruby, N. Dogra, M. Landini, R. Mottl, T. Donner, and T. Esslinger, Quantum Phases from Competing Short- and Long-Range Interactions in an Optical Lattice, Nature (London) 532, 476 (2016).

[15] L. W. Cheuk, M. A. Nichols, K. R. Lawrence, M. Okan, H. Zhang, E. Khatami, N. Trivedi, T. Paiva, M. Rigol, and M. W. Zwierlein, Observation of Spatial Charge and Spin Correlations in the 2d Fermi-Hubbard Model, Science 353, 1260 (2016).

[16] P. T. Brown, D. Mitra, E. Guardado-Sanchez, P. Schauß, S. S. Kondov, E. Khatami, T. Paiva, N. Trivedi, D. A. Huse, and W.S. Bakr, Spin-Imbalance in a $2 d$ Fermi-Hubbard System, Science 357, 1385 (2017).

[17] A. Mazurenko, C.S. Chiu, G. Ji, M. F. Parsons, M. Kansz-Nagy, R. Schmidt, F. Grusdt, E. Demler, D. Greif, and M. Greiner, A Cold-Atom Fermi-Hubbard Antiferromagnet, Nature (London) 545, 462 (2017).

[18] M. Cheneau, P. Barmettler, D. Poletti, M. Endres, P. Schausz, T. Fukuhara, C. Gross, I. Bloch, C. Kollath, and S. Kuhr, Light-Cone-Like Spreading of Correlations in a Quantum Many-Body System, Nature (London) 481, 484 (2012).

[19] A. de Paz, A. Sharma, A. Chotia, E. Maréchal, J. H. Huckans, P. Pedri, L. Santos, O. Gorceix, L. Vernac, and B. Laburthe-Tolra, Nonequilibrium Quantum Magnetism in a Dipolar Lattice Gas, Phys. Rev. Lett. 111, 185305 (2013).

[20] P. Richerme, Z.-X. Gong, A. Lee, C. Senko, J. Smith, M. Foss-Feig, S. Michalakis, A. V. Gorshkov, and C. Monroe, Non-local Propagation of Correlations in Quantum Systems with Long-Range Interactions, Nature (London) 511, 198 (2014).

[21] S. Hild, T. Fukuhara, P. Schauß, J. Zeiher, M. Knap, E. Demler, I. Bloch, and C. Gross, Far-from-Equilibrium Spin Transport in Heisenberg Quantum Magnets, Phys. Rev. Lett. 113, 147205 (2014).

[22] F. Meinert, M. J. Mark, E. Kirilov, K. Lauber, P. Weinmann, M. Grobner, A. J. Daley, and H.-C. Nagerl, Observation of Many-Body Dynamics in Long-Range Tunneling after a Quantum Quench, Science 344, 1259 (2014).

[23] P. Jurcevic, B. P. Lanyon, P. Hauke, C. Hempel, P. Zoller, R. Blatt, and C. F. Roos, Quasiparticle Engineering and Entanglement Propagation in a Quantum Many-Body System, Nature (London) 511, 202 (2014).

[24] J. Zhang, G. Pagano, P. W. Hess, A. Kyprianidis, P. Becker, H. Kaplan, A. V. Gorshkov, Z.-X. Gong, and C. Monroe, Observation of a Many-Body Dynamical Phase Transition with a 53-Qubit Quantum Simulator, Nature (London) 551, 601 (2017).

[25] A. Browaeys, D. Barredo, and T. Lahaye, Experimental Investigations of Dipole-Dipole Interactions between a Few Rydberg Atoms, J. Phys. B 49, 152001 (2016).

[26] P. Schauß, M. Cheneau, M. Endres, T. Fukuhara, S. Hild, A. Omran, T. Pohl, C. Gross, S. Kuhr, and I. Bloch, Observation of Mesoscopic Crystalline Structures in a Two-Dimensional Rydberg Gas, Nature (London) 491, 87 (2012).

[27] P. Schauss, J. Zeiher, T. Fukuhara, S. Hild, M. Cheneau, T. Macri, T. Pohl, I. Bloch, and C. Gross, Crystallization in Ising Quantum Magnets, Science 347, 1455 (2015).

[28] T. Pohl, E. Demler, and M. D. Lukin, Dynamical Crystallization in the Dipole Blockade of Ultracold Atoms, Phys. Rev. Lett. 104, 043002 (2010).

[29] D. Petrosyan, K. Mølmer, and M. Fleischhauer, On the Adiabatic Preparation of Spatially-Ordered Rydberg Excitations of Atoms in a One-Dimensional Optical Lattice by Laser Frequency Sweeps, J. Phys. B 49, 084003 (2016).

[30] H. Labuhn, D. Barredo, S. Ravets, S. de Léséleuc, T. Macrì, T. Lahaye, and A. Browaeys, Tunable Two-Dimensional Arrays of Single Rydberg Atoms for Realizing Quantum Ising Models, Nature (London) 534, 667 (2016).

[31] H. Bernien, S. Schwartz, A. Keesling, H. Levine, A. Omran, H. Pichler, S. Choi, A. S. Zibrov, M. Endres, M. Greiner, V. Vuletic, and M. D. Lukin, Probing Many-Body Dynamics on a 51-Atom Quantum Simulator, Nature (London) 551, 579 (2017).

[32] D. Barredo, S. de Léséleuc, V. Lienhard, T. Lahaye, and A. Browaeys, An Atom-by-Atom Assembler of DefectFree Arbitrary 2d Atomic Arrays, Science 354, 1021 (2016).

[33] The next-nearest-neighbor interaction strength is $(\sqrt{2})^{6}=8$ times smaller than the NN one and is, thus, one order of magnitude smaller than the Rabi frequency drive $\Omega$ and the inverse experimental timescale $1 / t_{\text {tot }}$.

[34] S. de Léséleuc, S. Weber, V. Lienhard, D. Barredo, H. P. Büchler, T. Lahaye, and A. Browaeys, Accurate Mapping of Multilevel Rydberg Atoms on Interacting Spin-1/2 Particles for the Quantum Simulation of Ising Models, Phys. Rev. Lett. 120, 113602 (2018). 
[35] S. Sachdev, Quantum Phase Transitions (Cambridge University Press, Cambridge, England, 2011), 2nd ed.

[36] H. W. J. Blöte and Y. Deng, Cluster Monte Carlo Simulation of the Transverse Ising Model, Phys. Rev. E 66, 066110 (2002).

[37] R. Moessner and S. L. Sondhi, Ising Models of Quantum Frustration, Phys. Rev. B 63, 224401 (2001).

[38] A. Das and B. K. Chakrabarti, Colloquium: Quantum Annealing and Analog Quantum Computation, Rev. Mod. Phys. 80, 1061 (2008).

[39] M. Schuler, S. Whitsitt, L.-P. Henry, S. Sachdev, and A. M. Läuchli, Universal Signatures of Quantum Critical Points from Finite-Size Torus Spectra: A Window into the Operator Content of Higher-Dimensional Conformal Field Theories, Phys. Rev. Lett. 117, 210401 (2016).

[40] C. Laumann, R. Moessner, A. Scardicchio, and S. Sondhi, Quantum Annealing: The Fastest Route to Quantum Computation?, Eur. Phys. J. Spec. Top. 224, 75 (2015).

[41] T. Caneva, M. Murphy, T. Calarco, R. Fazio, S. Montangero, V. Giovannetti, and G. E. Santoro, Optimal Control at the Quantum Speed Limit, Phys. Rev. Lett. 103, 240501 (2009).

[42] S. Deffner and S. Campbell, Quantum Speed Limits: From Heisenberg's Uncertainty Principle to Optimal Quantum Control, J. Phys. A 50, 453001 (2017).

[43] In order to improve the statistics, we symmetrize the experimental data for a given $(k, l)$ over the four quadrants $( \pm k, \pm l)$ for the square lattice (and in an adapted way for the triangular lattice). Since these operations are symmetries of the setup, the symmetrization does not alter genuine features in the experimental data.

[44] Using the $1 / r^{6}$ interaction would lead to finer structures around $x=0$ and $x=4$, but with very narrow steps in $x$.

[45] In the bulk or for $L$ even, the $\mathrm{AF}$ state is the equal superposition of the two classical Néel states. It has a uniform Rydberg density $n_{i}=1 / 2$, while $g^{(2)}(k, l)=$ $(-1)^{|k|+|l|} \times 1 / 4$. In stark contrast, on open boundary $L \times L$ arrays with $L$ odd, a single Néel ground state is selected. This state exhibits a staggered pattern of Rydberg densities, while the $g^{(2)}$ correlations vanish identically.

[46] We have checked that using NN interactions instead of the exact van der Waals coupling does not lead to appreciable changes in the dynamics.

[47] A. M. Läuchli and C. Kollath, Spreading of Correlations and Entanglement after a Quench in the One-Dimensional Bose-Hubbard Model, J. Stat. Mech. Theor. Exp. 2008, P05018 (2008).

[48] T. Langen, R. Geiger, M. Kuhnert, B. Rauer, and J. Schmiedmayer, Local Emergence of Thermal Correlations in an Isolated Quantum Many-Body System, Nat. Phys. 9, 640 (2013).

[49] K. Binder, High Temperature Expansions of Spin Correlation Functions for Ising and Heisenberg Ferromagnets, Phys. Status Solidi B 32, 891 (1969).

[50] G. Carleo, F. Becca, L. Sanchez-Palencia, S. Sorella, and M. Fabrizio, Light-Cone Effect and Supersonic Correlations in One- and Two-Dimensional Bosonic Superfluids, Phys. Rev. A 89, 031602 (2014).
[51] S. de Léséleuc, D. Barredo, V. Lienhard, A. Browaeys, and T. Lahaye, Analysis of Imperfections in the Coherent Optical Excitation of Single Atoms to Rydberg States, Phys. Rev. A 97, 053803 (2018).

[52] S. Humeniuk, Quantum Monte Carlo Study of Long-Range Transverse-Field Ising Models on the Triangular Lattice, Phys. Rev. B 93, 104412 (2016).

[53] A. M. Läuchli and R. Moessner, Quantum Simulations Made Easy Plane, arXiv:1504.04380.

[54] M. Hoening, W. Abdussalam, M. Fleischhauer, and T. Pohl, Antiferromagnetic Long-Range Order in Dissipative Rydberg Lattices, Phys. Rev. A 90, 021603 (2014).

[55] D. Barredo, H. Labuhn, S. Ravets, T. Lahaye, A. Browaeys, and C. S. Adams, Coherent Excitation Transfer in a Spin Chain of Three Rydberg Atoms, Phys. Rev. Lett. 114, 113002 (2015).

[56] S. de Léséleuc, D. Barredo, V. Lienhard, A. Browaeys, and T. Lahaye, Optical Control of the Resonant Dipole-Dipole Interaction between Rydberg Atoms, Phys. Rev. Lett. 119, 053202 (2017).

[57] A. P. Orioli, A. Signoles, H. Wildhagen, G. Günter, J. Berges, S. Whitlock, and M. Weidemüller, Relaxation of an Isolated Dipolar-Interacting Rydberg Quantum Spin System, Phys. Rev. Lett. 120, 063601 (2018).

[58] D. Peter, S. Müller, S. Wessel, and H. P. Büchler, Anomalous Behavior of Spin Systems with Dipolar Interactions, Phys. Rev. Lett. 109, 025303 (2012).

[59] E. Guardado-Sanchez, P. T. Brown, D. Mitra, T. Devakul, D. A. Huse, P. Schauß, and W. S. Bakr, preceding article, Probing the Quench Dynamics of Antiferromagnetic Correlations in a 2D Quantum Ising Spin System, Phys. Rev. X 8, 021069 (2018).

[60] D. Barredo, S. Ravets, H. Labuhn, L. Béguin, A. Vernier, F. Nogrette, T. Lahaye, and A. Browaeys, Demonstration of a Strong Rydberg Blockade in Three-Atom Systems with Anisotropic Interactions, Phys. Rev. Lett. 112, 183002 (2014).

[61] T. J. Park and J. C. Light, Unitary Quantum Time Evolution by Iterative Lanczos Reduction, J. Chem. Phys. 85, 5870 (1986).

[62] J. Dalibard, Y. Castin, and K. Mølmer, Wave-Function Approach to Dissipative Processes in Quantum Optics, Phys. Rev. Lett. 68, 580 (1992).

[63] R. Dum, P. Zoller, and H. Ritsch, Monte Carlo Simulation of the Atomic Master Equation for Spontaneous Emission, Phys. Rev. A 45, 4879 (1992).

[64] K. Mølmer, Y. Castin, and J. Dalibard, Monte Carlo WaveFunction Method in Quantum Optics, J. Opt. Soc. Am. B 10, 524 (1993).

[65] J. Johansson, P. Nation, and F. Nori, QuTiP 2: A Python Framework for the Dynamics of Open Quantum Systems, Comput. Phys. Commun. 184, 1234 (2013).

[66] M. B. Hastings, Locality in Quantum Systems, arXiv:1008 .5137 .

[67] L. Bonnes, F. H. L. Essler, and A. M. Läuchli, "LightCone” Dynamics after Quantum Quenches in Spin Chains, Phys. Rev. Lett. 113, 187203 (2014).

[68] P. Calabrese and J. Cardy, Time Dependence of Correlation Functions Following a Quantum Quench, Phys. Rev. Lett. 96, 136801 (2006). 
[69] P. Barmettler, D. Poletti, M. Cheneau, and C. Kollath, Propagation Front of Correlations in an Interacting Bose Gas, Phys. Rev. A 85, 053625 (2012).

[70] P. Hauke and L. Tagliacozzo, Spread of Correlations in Long-Range Interacting Quantum Systems, Phys. Rev. Lett. 111, 207202 (2013).
[71] S. Blanes, F. Casas, J. Oteo, and J. Ros, The Magnus Expansion and Some of Its Applications, Phys. Rep. 470, 151 (2009).

[72] J. Oitmaa, C. Hamer, and W. Zheng, Series Expansion Methods for Strongly Interacting Lattice Models (Cambridge University Press, Cambridge, England, 2006). 